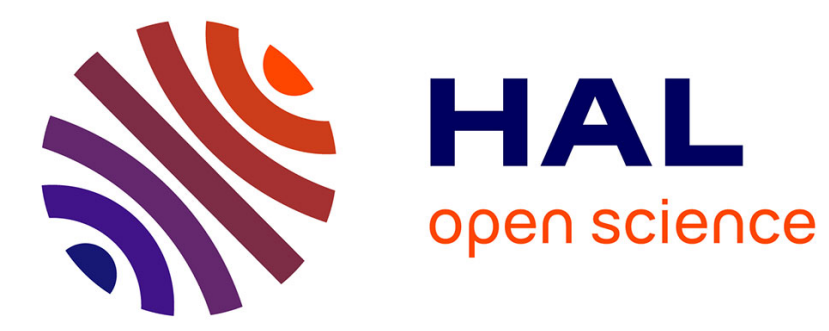

\title{
Elitism and Stochastic Dominance
}

Stephen Bazen, Patrick Moyes

\section{- To cite this version:}

Stephen Bazen, Patrick Moyes. Elitism and Stochastic Dominance. 2011. halshs-00576585

\section{HAL Id: halshs-00576585 \\ https://shs.hal.science/halshs-00576585}

Preprint submitted on 14 Mar 2011

HAL is a multi-disciplinary open access archive for the deposit and dissemination of scientific research documents, whether they are published or not. The documents may come from teaching and research institutions in France or abroad, or from public or private research centers.
L'archive ouverte pluridisciplinaire HAL, est destinée au dépôt et à la diffusion de documents scientifiques de niveau recherche, publiés ou non, émanant des établissements d'enseignement et de recherche français ou étrangers, des laboratoires publics ou privés. 


\section{GREQAM}

Groupement de Recherche en Economie Quantitative d'Aix-Marseille - UMR-CNRS 6579

Ecole des Hautes études en Sciences Sociales Universités d'Aix-Marseille II et III
Document de Travail

n$^{\circ} 2011-12$

\section{Elitism and Stochastic Dominance}

Stephen BAZEN

Patrick MOYES

March 2011 


\title{
Elitism and Stochastic Dominance*
}

\author{
Stephen Bazen ${ }^{\dagger}$ And PATrick Moyes ${ }^{\ddagger}$
}

* This paper forms part of the research project The Multiple Dimensions of Inequality (Contract No. ANR 2010 BLANC 1808) of the French National Agency for Research whose financial support is gratefully acknowledged. We are in particular indebted to Eugenio Peluso and Nicolas Gravel for helpful conversations and suggestions. We also would like to thank two anonymous referees whose comments have helped us to make our ideas more precise and to improve the paper. Needless to say, the authors bear the entire responsibility for remaining errors and deficiencies.

$\dagger$ GREQAM (UMR CNRS 6579), Université de la Méditerranée, Centre de la Vieille Charité, F-13002, Marseille, France. Email. stephen.bazen@univmed.fr.

$¥$ GREThA (UMR CNRS 5113), Université de Bordeaux, CNRS, Avenue Léon Duguit, F-33608, Pessac, and IDEP, Centre de la Vieille Charité, F-13002, Marseille, France. Email. patrick.moyes@u-bordeaux4.fr. 


\title{
Élitisme et Dominance Stochastique
}

\begin{abstract}
Résumé
La dominance stochastique est traditionellement associée à la mesure du risque et de l'inégalité et repose sur la concavité de la fonction d'utilité. Nous prétendons que l'approche en terme de dominance stochastique a des implications qui vont au-delà de la mesure du risque et de l'inégalité pour peu que l'on procède à certains ajustements. Nous appliquons ici la dominance stochastique à la mesure de l'élitisme, notion qui peut d'une certaine manière être considérée comme le contraire de l'égalitarisme. Alors que les critères de dominance stochastique habituels accordent plus de valeur aux distributions qui sont à la fois moins inégales et plus efficientes, nos critères garantissent qu'une distribution sera d'autant mieux classée qu'une autre qu'elle est à la fois plus efficiente et plus inégale. Afin d'illustrer notre approche, nous proposons deux exemples : (i) la comparaison d'une vingtaine de départements d'économie en Europe du point de vue de la performance scientifique, et (ii) la comparaison de différents pays sur la base de la notion d'affluence par opposition à celle de pauvreté.
\end{abstract}

Mots Clé : Fonction de Distribution Décumulative, Stochastic Dominance, Transfers Régressifs, Élitisme, Performance Scientifique, Affluence.

\section{Elitism and Stochastic Dominance}

\begin{abstract}
Stochastic dominance has been typically used with a special emphasis on risk and inequality reduction something captured by the concavity of the utility function in the expected utility model. We claim that the applicability of the stochastic dominance approach goes far beyond risk and inequality measurement provided suitable adaptations be made. We apply in the paper the stochastic dominance approach to the measurement of elitism which may be considered the opposite of egalitarianism. While the usual stochastic dominance quasi-orderings attach more value to more equal and more efficient distributions, our criteria ensure that, the more unequal and the more efficient the distribution, the higher it is ranked. Two instances are provided by (i) comparisons of scientific performance across institutions like universities or departments, and (ii) comparisons of affluence as opposed to poverty between countries.
\end{abstract}

Journal of Economic Literature Classification Number: D31, D63.

Keywords: Decumulative Distribution Functions, Stochastic Dominance, Regressive Transfers, Elitism, Scientific Performance, Affluence. 


\section{Introduction}

The stochastic dominance approach has been used in the areas of choice under uncertainty and inequality measurement for some time with a reasonable degree of success. A first advantage of this method of making comparisons is that all the distinct features that characterise the distributions under consideration are reflected in the ranking one obtains. Contrary to summary measures that aggregate the various characteristics of the distributions under review into a single index, the dominance criteria do not allow for such trade-offs. A second advantage is that the (partial) ranking one obtains is in accordance with a large spectrum of value judgements typically captured by the properties of the utility functions. By choosing ethically appealing properties for the utility functions and by requiring a consensus among the corresponding class of value judgements, one ends up with a ranking that is consistent with all the views reflected in this class. The properties that are considered relevant depend obviously on the question addressed and are open to debate. However it is a property common to all the stochastic dominance quasi-orderings that have been used up to now that modifications of the distributions that increase efficiency and equality improve welfare.

We claim that the applicability of the stochastic dominance approach is not limited to risk and inequality measurement, and that it can provide insights in other domains so long as suitable adjustments are made. In the income inequality literature it is assumed that the more equal the distribution is the better the society. Similarly, in the risk literature less risky prospects - that is lotteries whose outcomes are more concentrated around the expected value - are always preferable to riskier ones. There are however instances where it is not that clear whether a situation is considered superior as the result of a reduction of inequality or dispersion. In particular, we think of a concept like elitism which can be regarded as the opposite of egalitarianism. Let us make clear right from the beginning that we are not concerned here with the attitude of a group of people - like a caste - which is intent on defending privileges inherited from history or tradition. We rather have in mind the notion of excellence whether it is the result of innate talents or the attainment of characteristics giving rise to higher performance. We distinguish two different - even though they are not totally independent - ways of conceiving of elitism.

The first interpretation originates in the attitude according to which a person wants to be among the best (say among the top 5\%) in a particular domain such as music, sport, research, or politics, and does everything in order to achieve this objective. It is a personal quest of excellence originating in the individual's own preferences which may in turn be determined in part by her personal circumstances such as her family background or her socio-cultural environment. Then, one might be interested in measuring the overall extent of elitism in a particular dimension (income, intelligence, research performance) for the entire society. This evaluation necessitates that we decide (i) what is the threshold above which an individual can be considered an elitist in the particular dimension of interest, and (ii) what is the appropriate method of aggregating the individuals' achievements in terms of excellence to arrive at an overall measure of elitism in the society.

The second interpretation is policy oriented and it builds upon the idea that - because of technological constraints - it might be more efficient for the society to concentrate resources on the more talented of its members rather than distribute them evenly in the population. Elitism is then understood as the recognition that some groups of individuals deserve particular attention because they have specific skills, abilities, intellect, experience or wealth that are 
most likely to be constructive to society as a whole. To be more precise, it is a more effective arrangement from a social point of view to concentrate resources on the elite - those who are more talented - rather than on the less privileged. Greater inequality of resources which in this context is regarded as a transfer of resources from the less endowed to the more endowed - is a means of achieving higher performance which in turn may be of benefit to all. Elitist policies are frequently invoked in areas such as education, technology, innovation or scientific research, and always in connection with the pursuit of greater economic performance. This leaves open the questions to know how efficiency and talents are defined and measured. This approach necessitates among other things that one (i) identifies the factors (technology, preferences) that explain why a more unequal allocation of resources in favour of the more talented gives rise to a better outcome, and (ii) specifies what is meant by a more effective arrangement - one may think for instance of the possibility of compensating the disadvantaged - that justifies such a position. ${ }^{1}$

The first conception of elitism is a purely descriptive approach which aims at deriving an overall measure of excellence in the society starting with the individuals' achievements in terms of excellence. To this extent, it is reminiscent of the way one typically thinks of poverty measurement in the economic literature: one first identifies the poor population and then one aggregates the deprivations of the poor to get an overall measure of poverty. It is immaterial whether the variable of interest is a factor contributing to (e.g. talent) or resulting from (e.g. income) the production process. On the contrary, the second conception of elitism involves a behavioural model where agents make decisions on the basis of their preferences but where their decisions have differing impacts on the economic outcome due to the fact that they have different talents. ${ }^{2}$ We are interested here in the measurement of elitism and we do not address the important question of knowing under which circumstances elitist policies prove to be socially more effective than egalitarian policies, even though this important political issue is in the background.

As a matter of illustration, we consider two examples where the concern for elitism has given rise to conflicting views in the public and among politicians. Our first example is related to the question of what is the most effective way for increasing a society's well-being: focusing on the poor and implementing transfers to improve their situations or designing policies in order to increase affluence in the society. Following Sen's influential contribution (see e.g. Sen (1976), Sen (1992, Chapter 7)), there is considerable interest in the impact on poverty of changes in the shape of the distribution of personal income. It is typically admitted that the alleviation of poverty is a main objective in modern societies in order to achieve greater well-being. This is mirrored in the discussion among politicians and the public about a more affluent society which is seen as synonymous with a reduction in poverty. However, this assumes that affluence and poverty are just two sides of the same coin and that dominance of one distribution over another is associated with greater affluence. The connection between stochastic dominance criteria and the measurement of poverty has long been recognised (see e.g. Foster and Shorrocks (1988)). If one distribution stochastically dominates another, then poverty is unambiguously less in the former distribution than in the latter, and conversely.

1 The simplest model one may think of would consist of (i) a planner maximising a symmetric, monotone and concave function of the society's members' utilities, (ii) a production process where the more talented always produce more with the same resources than the less talented, and (iii) a costless ex-post redistribution process

${ }^{2}$ Considering an artisan economy with no taxation, Ebert and Moyes (2010) have investigated the impact on the income distribution of particular changes in the allocation of talents among agents. 
Does that mean that a decrease in poverty as measured by stochastic dominance is always equivalent to a decrease in affluence? Actually this is not necessarily the case as we will show below unless the distributions under consideration have equal means, which rules out the potential conflict between equality and efficiency.

A second example in which elitism would seem to be particularly relevant is the measurement of the scientific performance of academics and institutions in terms of research which is now common practice in most countries. Articles, taken as an evidence of research output, are given values that may depend among other things on the ranking and impact factors of the journals where they are published or on the number of citations they receive. Our starting point is the recognition that every evaluation process involves value judgements whether they are explicit or not. The adoption of one set of value judgements or another generally leads to different rankings in terms of scientific performance. It is therefore important to understand the value judgements contained implicitly in the measures used when proceeding to the evaluation. A routinely used index may possess properties that make it unsuitable for making comparisons of scientific performance. Also universities, departments, research centers or academics are expected to implement - possibly conflicting - strategies in response to the way their scientific performance is evaluated. A researcher is generally considered to be active if her output is satisfactory in a quantitative sense. However, scholars establish their individual reputation by having their articles published in the top-ranked journals. There may indeed be a conflict between individuals' publishing strategies and the objectives of their research institute or department. An examination of the literature reveals that, with a few exceptions, most studies compute summary statistics in order to compare the scientific performance of institutions and academics (see among others Combes and Linnemer (2003) for France, Kalaitzidakis, Mamuneas, and Stengos (1999, 2003) and Lubrano, Bauwens, Kirman, and Protopopescu (2003) for Europe, Dusansky and Vernon (1998) for the US, and Coupé (2003) for the World). It is true that some authors have examined distribution of the scientific output of academic institutions. Lubrano and Protopopescu (2004) compare seven European countries using statistical estimates of density function of publication scores of economics departments and apply stochastic dominance techniques to rank countries. Unlike the present paper, their approach is statistical and is not based on explicit normative foundations. An unpublished paper by Carayol and Lahatte (2009) also takes into account the distributional aspect of scientific output in a comparison of French academic institutions in all disciplines other than humanities and social sciences. The criteria they use to compare institutions are very different from those that arise from the normative foundations based on the notion of elitism.

In this paper we suggest that the technique of stochastic dominance can be used to compare the degree of elitism - be it in terms of income or publication scores - of different societies. This of course calls for an adaptation of the properties of the utility function or as we call it, the value function - retained for assessing the contribution of the members in a society. It is reasonable to maintain that the value function is non-decreasing with an individual's contribution as in the standard stochastic dominance approach. However, while a more equal distribution is always considered a desirable thing under standard stochastic dominance, elitism carries the idea that more concentration at the top of the scale results in a social improvement. Formally this can be conceived in terms of a regressive transfer that consists in increasing the contribution of the more endowed at the expense of less endowed individuals. Whereas the concavity of the utility function guarantees that social welfare - as measured by the sum of utilities - decreases as the result of a regressive transfer, it is claimed 
that elitism increases when the value function is convex. Because convexity is the opposite of concavity, it is tempting to appeal to standard second degree stochastic dominance and declare that the performance of society A is superior to that of society B if A second degree stochastic dominates B. However, this is not the case because the conditions we impose - namely that performance increases as the result of increments and regressive transfers - come into conflict under standard second degree stochastic dominance. Substituting the survival function - or the decumulative distribution function as we call it in the paper - for the cumulative distribution function will resolve the difficulty. Then, it can be shown that one distribution generates greater elitism than another whatever the value function provided it is increasing and convex if and only if the integral of the survival function of the first distribution is nowhere below that of the second distribution. Similarly, convexity of the derivative of the value function is both necessary and sufficient for performance to increase when a regressive transfer among the more talented is coupled with a progressive transfer among less talented individuals in such a way that the variance is preserved. The quasi-orderings we obtain are shown to depart significantly from the standard stochastic dominance criteria even though they are admittedly very close in spirit.

We present in Section 2 our conceptual framework and argue in favour of a consensual approach for comparing societies from the point of view of elitism. We introduce in Section 3 different classes of value functions that are believed to capture essential dimensions of the achievements of a society and we identify the corresponding dominance criteria. As a matter of illustration, we apply our criteria in Section 4 to the comparison of affluence across different countries and to the comparison of European economics departments in terms of scientific performance and we contrast the rankings we get with those arising from the application of standard stochastic dominance. We conclude in Section 5 and sketch the proofs of the results in Section 6.

\section{The Framework}

We are interested in the comparisons of different societies from the point of view of their performance where the latter is determined by the distribution of some cardinal attribute. These societies can be seen as economies, universities, departments, or research centers. The attribute, whose distribution we are interested in, may be income, publication scores, talents or the results of intelligence tests. We adopt a discrete framework and we associate a society with its attribute profile $\mathbf{x}:=\left(x_{1}, \ldots, x_{n(x)}\right)$, where $x_{i}$ indicates the endowment of individual $i$ in the attribute and where $n(\mathbf{x})$ is the dimension of the profile $\mathbf{x}$. We assume throughout that $n(\mathbf{x}) \geqq 3$ and $x_{i} \in D$, where $D=[\underline{s}, \bar{s}] \subset \mathbb{R}$. Because all the criteria we will consider below for making comparisons in terms of elitism are symmetric - a permutation of endowments within the societies does not change the results - we henceforth suppose without loss of generality that the individuals' endowments are arranged in the non-decreasing order. We use $\mathscr{S}_{n}(D)$ to represent the set of profiles involving $n$ individuals and let

$$
\mathscr{S}(D):=\bigcup_{n=3}^{+\infty} \mathscr{S}_{n}(D)
$$

represent the general set of profiles. The arithmetic mean of profile $\mathbf{x} \in \mathscr{S}(D)$ is denoted as $\mu(\mathbf{x}):=\sum_{i=1}^{n(\mathbf{x})} x_{i} / n(\mathbf{x})$. The density function of $\mathbf{x} \in \mathscr{S}(D)$ is indicated by $f(s ; \mathbf{x}):=$ $n(s ; \mathbf{x}) / n(\mathbf{x})$, for all $s \in(-\infty,+\infty)$, where $n(s ; \mathbf{x}):=\#\left\{i \in\{1,2, \ldots, n(\mathbf{x})\} \mid x_{i}=s\right\}$. We denote as $F(\cdot ; \mathbf{x})$ the cumulative distribution function (cdf) of $\mathbf{x} \in \mathscr{S}(D)$ defined by $F(s ; \mathbf{x})$ : $=q(s ; \mathbf{x}) / n(\mathbf{x})$, for all $s \in(-\infty,+\infty)$, where $q(s ; \mathbf{x}):=\#\left\{i \in\{1,2, \ldots, n(\mathbf{x})\} \mid x_{i} \leqq s\right\}$. We 
let $Q(\cdot ; \mathbf{x})$ represent the inverse cumulative distribution function (icdf) - or equivalently, the quantile function - of $\mathbf{x}$ obtained by letting $Q(0 ; \mathbf{x}):=x_{1}$ and

$$
Q(p ; \mathbf{x}):=\inf \{s \in(-\infty,+\infty) \mid F(s ; \mathbf{x}) \geqq p\}, \forall p \in(0,1]
$$

(see Gastwirth (1971)). It is convenient to introduce the decumulative distribution function (ddf) - also known as the survival function - of $\mathbf{x} \in \mathscr{S}(D)$ defined by $D F(s ; \mathbf{x}):=1-F(s ; \mathbf{x})$, for all $s \in(-\infty,+\infty)$. Letting $r(s ; \mathbf{x}):=\#\left\{i \in\{1,2, \ldots, n(\mathbf{x})\} \mid x_{i}>s\right\}$, we note that $D F(s ; \mathbf{x}):=r(s ; \mathbf{x}) / n(\mathbf{x})$, for all $s \in(-\infty,+\infty)$. We indicate by $R Q(p ; \mathbf{x})$ the inverse decumulative distribution function (iddf) - or equivalently, the reverse quantile function - of $\mathbf{x}$ defined by $R Q(1 ; \mathbf{x}):=x_{1}$ and

$$
R Q(p ; \mathbf{x}):=\sup \{s \in(-\infty,+\infty) \mid D F(s ; \mathbf{x}) \leqq p\}, \forall p \in[0,1) .
$$

It follows from the definition of the reverse quantile function that $R Q(p ; \mathbf{x})=Q(1-p ; \mathbf{x})$, for all $\mathbf{x} \in \mathscr{S}(D)$ and all $p \in[0,1]$.

We follow the utilitarian - or equivalently, the expected utility - tradition and we assume that the performance of a society with profile $\mathbf{x} \in \mathscr{S}(D)$ is given by

$$
W_{v}(\mathbf{x}):=\frac{1}{n(\mathbf{x})} \sum_{i=1}^{n(\mathbf{x})} v\left(x_{i}\right) \equiv \int_{\underline{s}}^{\bar{s}} v(s) f(s ; \mathbf{x}) d s,
$$

where $v$ is the value function defined up to an increasing affine transformation. We assume that the value function $v$ is continuous and differentiable up to the third order and we indicate by $\mathscr{V}(D)$ such a set. We refer the reader to Fishburn and Vickson (1978) for a rigorous justification of this way of proceeding. Given two profiles $\mathbf{x}, \mathbf{y} \in \mathscr{S}(D)$, we will say that profile $\mathbf{x}$ is a replication of profile $\mathbf{y}$ if there exists an integer $r \geqq 2$ such that $\mathbf{x}=\left(\mathbf{y}^{1} ; \ldots ; \mathbf{y}^{n(\mathbf{y})}\right)$, where $\mathbf{y}^{i}:=\left(y_{i}, \ldots, y_{i}\right) \in D^{r}$, for all $i=1,2, \ldots, n(\mathbf{y})$, and $n(\mathbf{x})=r n(\mathbf{y})$. It follows directly from definition (2.4) that performance is not modified by a replication: $W_{v}(\mathbf{x})=W_{v}(\mathbf{y})$ whenever $\mathbf{x}$ is a replication of $\mathbf{y}$. Before we proceed further we would like to insist on the fact that we are interested in the ranking of profiles in terms of performance. This means among other things that we prevent ourselves from concluding that profile $\mathbf{x}$ does twice as much as profile $\mathbf{y}$ in terms of performance if it happens that $W_{v}(\mathbf{x})=2 W_{v}(\mathbf{y})$. Similarly we resist making the judgement that the difference in performance between profiles $\mathbf{x}$ and $\mathbf{y}$ is greater than the difference in performance between profiles $\mathbf{z}$ and $\mathbf{t}$ because $W_{v}(\mathbf{x})-W_{v}(\mathbf{y})>$ $W_{v}(\mathbf{z})-W_{v}(\mathbf{t})$. This means that societal performance is measured on an ordinal scale and that any increasing transformation of $W_{v}(\cdot)$ provides the same information as does the original performance function. For instance, choosing

$$
\Xi_{v}(\mathbf{x}):=v^{-1}\left(\frac{1}{n(\mathbf{x})} \sum_{i=1}^{n(\mathbf{x})} v\left(x_{i}\right)\right)
$$

as a measure of performance would not modify the ranking of profiles implied by (2.4). This measure of performance is reminiscent of what is known as the equally distributed income in the inequality literature.

The value function $v$ captures the attitude of an observer - be it the policy maker, either author of this paper or anyone who is willing to play this role - concerning the way the distribution of the attribute among the society's members determines its performance. Clearly, choosing an arbitrary value function $v \in \mathscr{V}(D)$ results in a particular ordering of the profiles 
under comparison. For instance, substituting $v(s)=s$ into (2.5) amounts to measuring the performance of a profile by the arithmetic mean, while taking $v(s)=\ln s$ would result in the geometric mean. Having the evaluation of performance in mind we expect that certain value functions are more appropriate than others. Before turning to the question of which specific properties of the value function are deemed relevant when one is interested in comparing performance across profiles, we first suggest a strategy that avoids relying exclusively on a particular value function. Suppose that the subclass $\mathscr{V}^{*}(D)$ of possible value functions consists of all those value functions that are relevant for measuring the contribution of an individual to the performance of the society. Then, we will say that profile $\mathbf{x}$ does unanimously no worse in terms of performance than profile $\mathbf{y}$, which we write $\mathbf{x} \geq_{\mathscr{V} *} \mathbf{y}$, if

$$
W_{v}(\mathbf{x}) \geqq W_{v}(\mathbf{y}), \forall v \in \mathscr{V}^{*}(D) .
$$

The obvious question that will occupy us in the next section is to know (i) what this partial ordering of the profiles under comparison would look like, and (ii) how it is altered as the result of changes in the class of relevant value functions.

\section{Three Dominance Criteria}

We claimed above that the assessment of a society's performance is a normative exercise that involves value judgements. The first stage in this assessment process is to choose the overall performance evaluation function which we assume here is of the utilitarian type defined by (2.4) and is given once-and-for-all. The second stage is to determine the properties of the value function that are deemed desirable. We follow the traditional practice in welfare economics which consists (i) in suggesting elementary transformations of the profiles that are expected to improve performance, and (ii) in identifying the restrictions they impose on the value function for performance as measured by (2.4) to increase. Before we address these questions, we would like to introduce an example that we will invoke repeatedly in order to illustrate various points.

EXAMPLE 3.1. Consider the three following profiles which for simplicity have the same number of individuals: $\mathbf{x}^{1}=(1,4,5,8), \mathbf{x}^{2}=(2,3,5,6)$ and $\mathbf{x}^{3}=(2,4,5,7)$. We note incidently that, if one chooses the arithmetic mean as the index of performance, then profiles $\mathbf{x}^{1}$ and $\mathbf{x}^{3}$ are equivalent and rank above profile $\mathbf{x}^{2}$.

We are confident that nobody would object to the judgement that performance increases - or at least does not decrease - when, other things equal, the endowment of one individual increases. Given two profiles $\mathbf{x}, \mathbf{y} \in \mathscr{S}(D)$ with $n(\mathbf{x})=n(\mathbf{y})$, we will say that profile $\mathbf{x}$ is obtained from profile $\mathbf{y}$ by means of an increment if there exists $\Delta>0$ and an individual $i$ such that

$$
x_{i}=y_{i}+\Delta \text { and } x_{j}=y_{j}, \forall j \neq i \text {. }
$$

Examination of Example 3.1 reveals that profile $\mathbf{x}^{2}$ is converted into profile $\mathbf{x}^{3}$ by means of two increments and that there are no other cases where one profile can be obtained from another by means of increments. It does not take long to recognise that performance as measured by (2.4) improves as the result of an increment if and only if the value function $v$ is non-decreasing. We indicate by

$$
\mathscr{V}_{1}(D):=\left\{v \in \mathscr{V}(D) \mid v^{\prime}(s) \geqq 0, \forall s\right\}
$$


the class of value functions that are non-decreasing. It is therefore natural to appeal to value functions in the class $\mathscr{V}_{1}(D)$ in order to evaluate the performance of different societies. However, the fact that elements in the class $\mathscr{V}_{1}(D)$ all have in common that they record increments as improvements of a society's performance does not imply that they agree on the ranking of the societies under comparison. Consider for instance the value functions $v^{\circ}(s)=s^{2}$ and $v^{*}(s)=\sqrt{s}$ that are both increasing and belong to $\mathscr{V}_{1}(D)$. Then, we obtain that $W_{v^{\circ}}\left(\mathbf{x}^{1}\right)>W_{v^{\circ}}\left(\mathbf{x}^{3}\right)$ while $W_{v^{*}}\left(\mathbf{x}^{1}\right)<W_{v^{*}}\left(\mathbf{x}^{3}\right)$. It is interesting to investigate in which circumstances one would get the same ranking of two arbitrary profiles $\mathbf{x}$ and $\mathbf{y}$ irrespective of the choice of a value function provided it be non-decreasing. The unanimity condition (2.6) with $\mathscr{V}^{*}(D)=\mathscr{V}_{1}(D)$ precisely avoids conflicting views regarding the choice of the value function. However seductive such a criterion is, the difficulty is that it is impossible to apply it in practice as it requires an infinite number of pairwise comparisons to be made in order to decide when one profile is unambiguously better than another. There is therefore a need for practical procedures that will allow one to check whether condition (2.6) holds or not, when $\mathscr{V}^{*}(D)=\mathscr{V}_{1}(D)$. The following result, which is a straightforward adaptation of standard results in the risk and inequality literature (see e.g. Saposnik (1981), Fishburn and Vickson (1978)), proposes two such procedures in order to implement unanimity over the class of non-decreasing value functions.

Theorem 3.1. Let $\mathbf{x}, \mathbf{y} \in \mathscr{S}(D)$. The following three statements are equivalent:

(a) $W_{v}(\mathbf{x}) \geqq W_{v}(\mathbf{y})$, for all $v \in \mathscr{V}_{1}(D)$.

(b) $D F(s ; \mathbf{x}) \geqq D F(s ; \mathbf{y})$, for all $s \in[\underline{s}, \bar{s}]$.

(c) $R Q(p ; \mathbf{x}) \geqq R Q(p ; \mathbf{y})$, for all $p \in[0,1]$.

Statement (b) of Theorem 3.1, which involves the decumulative distribution functions - or equivalently the survival function - of profiles $\mathbf{x}$ and $\mathbf{y}$, defines first order decumulative stochastic dominance. More precisely, we will say that profile $\mathbf{x}$ first order decumulative stochastic dominates profile $\mathbf{y}$ if the percentage of individuals whose endowments in $\mathbf{x}$ are greater or equal to $s$ is not less than the corresponding percentage in $\mathbf{y}$, whatever the value $s$ takes in the interval $[\underline{s}, \bar{s}]$. Statement (c) involves the quantiles of $\mathbf{x}$ and $\mathbf{y}$ but arranged in reverse order of endowments - from top to bottom - and we will say that profile $\mathbf{x}$ reverse quantile dominates profile $\mathbf{y}$ when it holds. At first sight, statements (b) and (c) of Theorem 3.1 depart somewhat from the conditions one encounters in the standard first order stochastic dominance results. However, since $D F(s ; \mathbf{x})-D F(s ; \mathbf{y}) \geqq 0$ implies $F(s ; \mathbf{x})-F(s ; \mathbf{y}) \leqq 0$, for all $s \in[\underline{s}, \bar{s}]$, for first order dominance it does not make any difference whether we use the cumulative or decumulative distribution functions. Nor does the ranking of profiles change when one appeals to the quantile and reverse quantile functions. As we will see in a while, things appear to be different when we move to dominance criteria of higher orders, unless one imposes special restrictions on the profiles under comparison.

The monotonicity of the value function ensures that the performance attached to a profile improves as soon as one individual experiences an increase in her endowment irrespective of her relative position on the attribute scale. From the point of view of performance it makes sense to attach more value to increments affecting individuals who rank at the top rather than at the bottom of the scale. Put differently, the more concentrated endowments are at the top of the attribute scale, the greater the performance of the society. This idea is formally captured by the concept of a mean preserving spread in the risk literature. Here we borrow the 
approach of the inequality literature and, given two profiles $\mathbf{x}, \mathbf{y} \in \mathscr{S}(D)$ with $n(\mathbf{x})=n(\mathbf{y})$, we will say that profile $\mathbf{x}$ is obtained from profile $\mathbf{y}$ by means of a regressive transfer if there exists $\Delta>0$ and two individuals $i$ and $j$ such that

$$
\begin{aligned}
& y_{h}=x_{h}, \forall h \neq i, j ; \\
& y_{i}=x_{i}+\Delta ; y_{j}=x_{j}-\Delta ; \\
& \Delta \leq\left(x_{j}-x_{i}\right) / 2 .
\end{aligned}
$$

We note that by definition a regressive transfer leaves the mean of the distribution unchanged: $\mu(\mathbf{x})=\mu(\mathbf{y})$. A regressive transfer consists in increasing the endowment of a highly ranked individual at the expense of a decrease of the endowment of a lower ranked individual so long as their relative positions remain unaltered. Going back to Example 3.1, we note that profile $\mathbf{x}^{1}$ is obtained from profile $\mathbf{x}^{3}$ by means of a regressive transfer of one unit involving the first and fourth individuals while one increment and two regressive transfers are needed to transform $\mathbf{x}^{2}$ into $\mathbf{x}^{1}$ It can be easily seen that convexity of the value function is both necessary and sufficient for performance to increase as the result of a regressive transfer. Consider two profiles $\mathbf{x}$ and $\mathbf{y}$ verifying condition (3.3), hence $\mathbf{x}$ is derived from $\mathbf{y}$ by means of a regressive transfer. Then, $W_{v}(\mathbf{x}) \geqq W_{v}(\mathbf{y})$ is equivalent to

$$
v\left(y_{i}\right)-v\left(x_{i}\right) \leqq v\left(x_{j}\right)-v\left(y_{j}\right) .
$$

Upon integrating, condition (3.4) reduces to

$$
\int_{0}^{\Delta} v^{\prime}\left(x_{i}+s\right) d s \leqq \int_{0}^{\Delta} v^{\prime}\left(y_{j}+s\right) d s
$$

which, since $x_{i}<y_{j}$, holds if $v^{\prime}(s)$ is non-decreasing with $s$. That the latter condition is also necessary for performance to increase as the result of a regressive transfer is readily seen. For suppose $v$ is not convex, in which case there exist $s, t \in D$ with $s<t$ such that $2 v((s+t) / 2)>v(s)+v(t) .{ }^{3}$ Choosing for instance $\mathbf{x}=(s, t)$ and $\left.\mathbf{y}=((s+t) / 2,(s+t) / 2)\right)$, we obtain $W_{v}(\mathbf{x})<W_{v}(\mathbf{y})$. We indicate by

$$
\mathscr{V}_{2}(D):=\left\{v \in \mathscr{V}(D) \mid v^{\prime}(s) \geqq 0 \text { and } v^{\prime \prime}(s) \geqq 0, \forall s\right\}
$$

the class of non-decreasing and convex value functions. Because $\mathscr{V}_{2}(D) \subset \mathscr{V}_{1}(D)$, the application of unanimity over the class $\mathscr{V}_{2}(D)$ will result in a finer ranking of the profiles under comparison than the one that would have obtained from the application of the same principle to the class $\mathscr{V}_{1}(D)$. But this does not provide much guidance on the nature of the procedures that enable dominance to be detected with the exception that they will be consistent with conditions (b) and (c) of Theorem 3.1. The following result, which presents the procedures for implementing unanimous agreement for all non-decreasing and convex value functions, is not that surprising.

Theorem 3.2. Let $\mathbf{x}, \mathbf{y} \in \mathscr{S}(D)$. The following three statements are equivalent:

(a) $W_{v}(\mathbf{x}) \geqq W_{v}(\mathbf{y})$, for all $v \in \mathscr{V}_{2}(D)$.

(b) $\int_{s}^{\bar{s}} D F(t ; \mathbf{x}) d t \geqq \int_{s}^{\bar{s}} D F(t ; \mathbf{y}) d t$, for all $s \in[\underline{s}, \bar{s}]$.

(c) $\int_{0}^{p} R Q(\xi ; \mathbf{x}) d \xi \geqq \int_{0}^{p} R Q(\xi ; \mathbf{y}) d \xi$, for all $p \in[0,1]$.

3 This argument exploits the continuity of the value function $v$ : for a justification see for instance Hardy, Littlewood, and Pólya (1952, Chapter 3). 
Figure 3.1: Integrals of the cumulative distribution functions of $\mathbf{x}^{1}, \mathbf{x}^{2}$ and $\mathbf{x}^{3}$

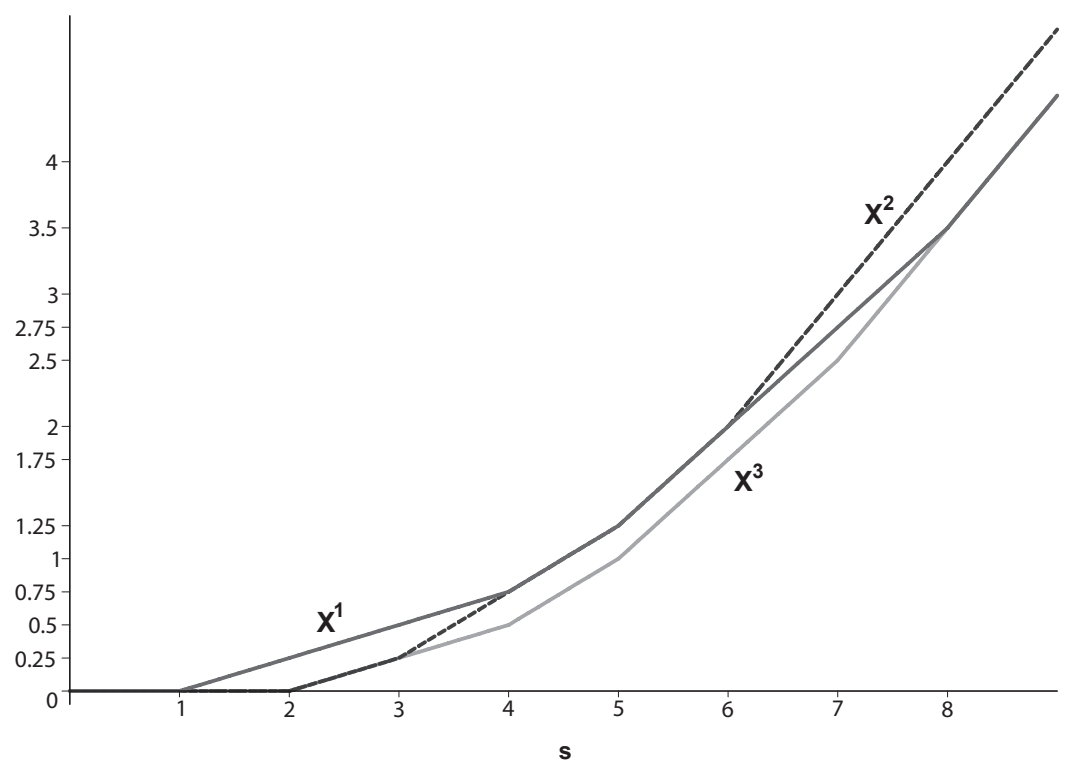

Figure 3.2: Integrals of the decumulative distribution functions of $\mathbf{x}^{1}, \mathbf{x}^{2}$ and $\mathbf{x}^{3}$

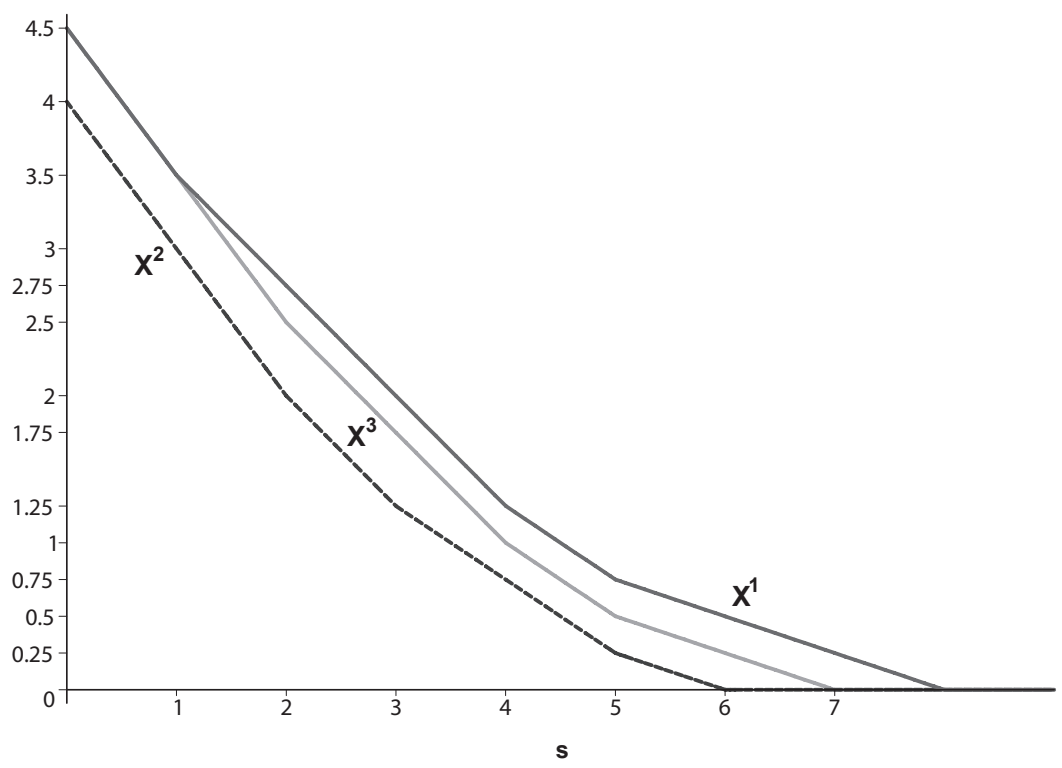

Statement (b) of Theorem 3.2 defines second order decumulative stochastic dominance: if and only if the integral of the decumulative distribution function of profile $\mathbf{x}$ is nowhere below that of profile $\mathbf{y}$ will we say that $\mathbf{x}$ second order decumulative stochastic dominates $\mathbf{y}$. Similarly, we will say that profile $\mathbf{x}$ reverse generalised Lorenz dominates profile $\mathbf{y}$ if its reverse generalised Lorenz curve lies nowhere below that of $\mathbf{y}$, which is statement (c) of Theorem 
Figure 3.3: Generalised Lorenz curves of $\mathbf{x}^{1}, \mathbf{x}^{2}$ and $\mathbf{x}^{3}$

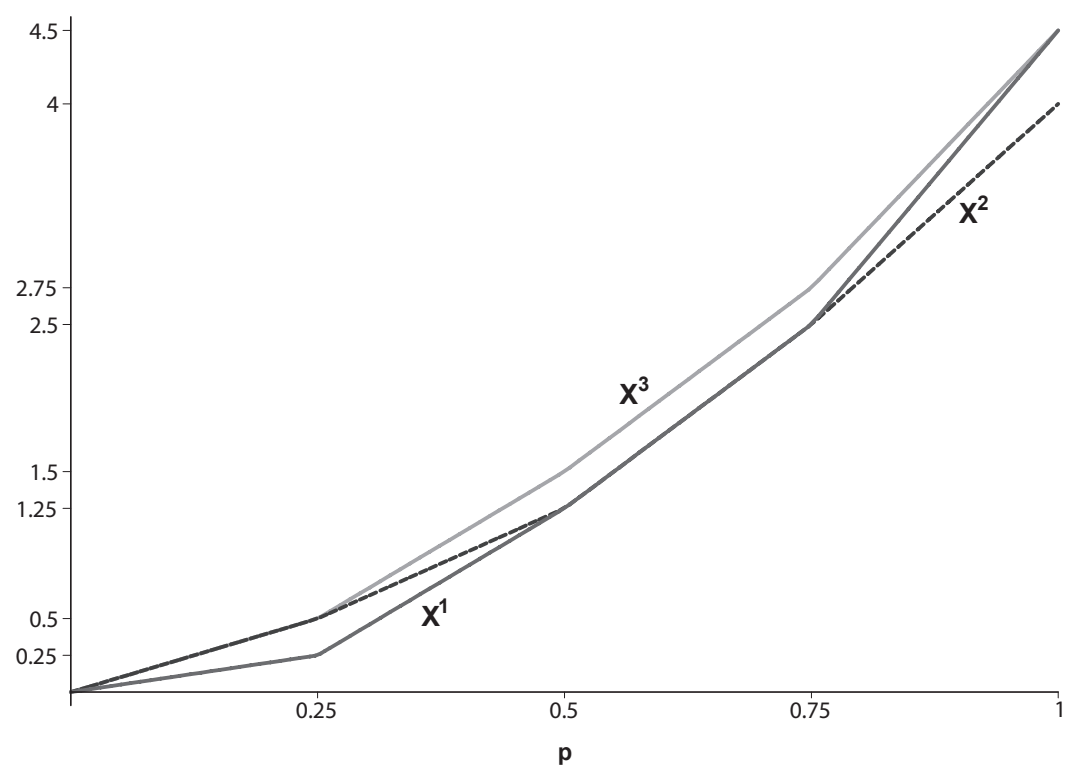

Figure 3.4: Reverse generalised Lorenz curves of $\mathbf{x}^{1}, \mathbf{x}^{2}$ and $\mathbf{x}^{3}$

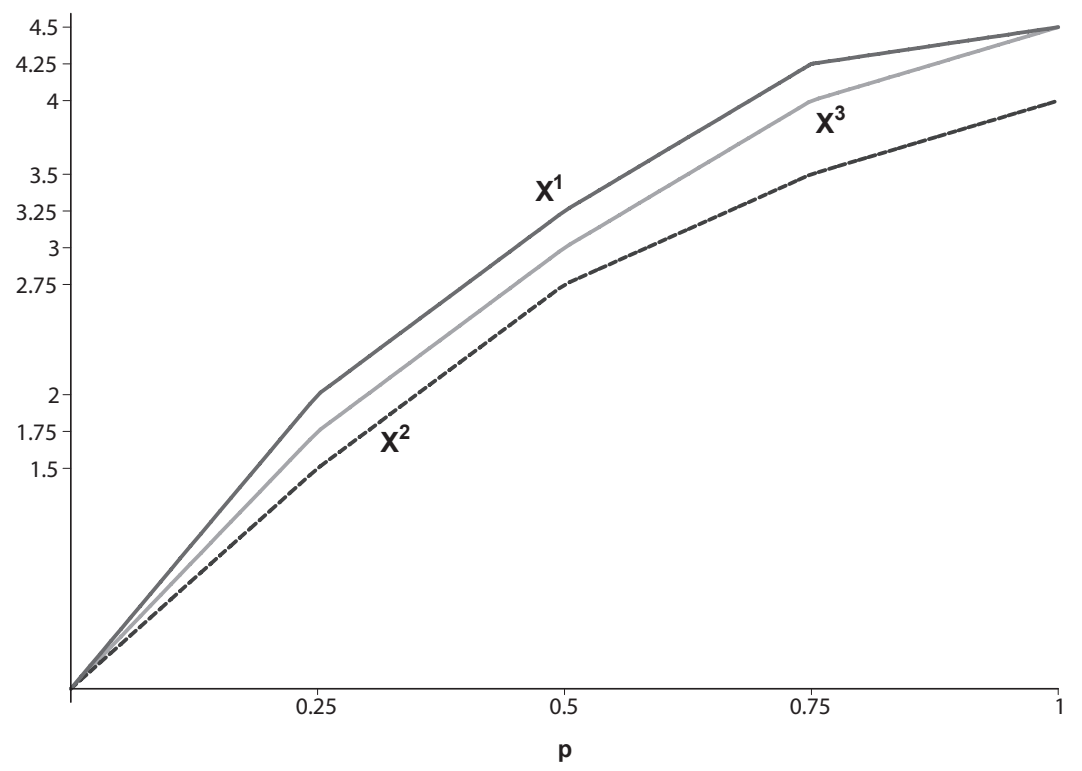

3.2. We should first emphasise the distinction between standard second degree stochastic dominance and statement (b) of Theorem 3.2. Second degree stochastic dominance requires that the cumulative distribution functions be integrated from $\underline{s}$ up to $s$. In our case it is the decumulative distribution functions that have to be integrated and integration must be performed from $s$ up to $\bar{s}$. Contrary to what is the case in Theorem 3.1, the application of 
standard second order stochastic dominance procedures and the criterion defined by condition (b) in Theorem 3.2 generally lead to differing rankings of the profiles. Actually, the rankings of profiles generated by these two procedures coincide only when the profiles have equal means (see Section 6, Proposition 6.1). This is illustrated in Figures 3.1 and 3.2 where we have plotted the integrals of the cumulative and decumulative distribution functions of the profiles of Example 3.1. A similar observation has to be made concerning the distinction between generalised Lorenz dominance and reverse generalised Lorenz dominance. The first criterion requires that the quantile curves be integrated from 0 up to $p$ and it declares that profile $\mathbf{x}$ generalised Lorenz dominates profile $\mathbf{y}$, which is written as $\mathbf{x} \geq_{G L} \mathbf{y}$, if and only if

$$
\int_{0}^{p} Q(\xi ; \mathbf{x}) d \xi \geqq \int_{0}^{p} Q(\xi ; \mathbf{y}) d \xi, \forall p \in[0,1]
$$

(see Shorrocks (1983)). As condition (c) of Theorem 3.2 makes clear, it is the reverse quantile functions that have to be integrated from 0 up to $p$ in the case of reverse generalised Lorenz dominance. The generalised Lorenz curves and the reverse generalised Lorenz curves are depicted respectively in Figures 3.3 and 3.4. We also note that, though a higher mean is a necessary condition for second degree decumulative stochastic domination (see Section 6), it is by no means sufficient as it can be easily verified. Indeed choose $\mathbf{x}^{4}=(3,3,7), \mathbf{x}^{5}=$ $(1,5,6)$, which implies that $\mu\left(\mathbf{x}^{4}\right)=\frac{13}{3}>\frac{12}{3}=\mu\left(\mathbf{x}^{5}\right)$. Inspection of Figure 3.5 indicates that condition (b) of Theorem 3.2 does not hold. We know that by definition of generalised Lorenz dominance, $\min \left\{x_{i}\right\} \geqq \min \left\{y_{i}\right\}$ is a necessary condition for $\mathbf{x}$ to generalised Lorenz or equivalently second degree stochastic - dominate $\mathbf{y}$. It is not surprising that $\max \left\{x_{i}\right\} \geqq$ $\max \left\{y_{i}\right\}$ is necessary for $\mathbf{x}$ to be ranked above $\mathbf{y}$ by the reverse generalised Lorenz criterion. Conditions (b) and (c) - and as a consequence condition (a) - above incorporate a concern for

Figure 3.5: Integrals of the decumulative distribution functions of $\mathbf{x}^{4}$ and $\mathbf{x}^{5}$

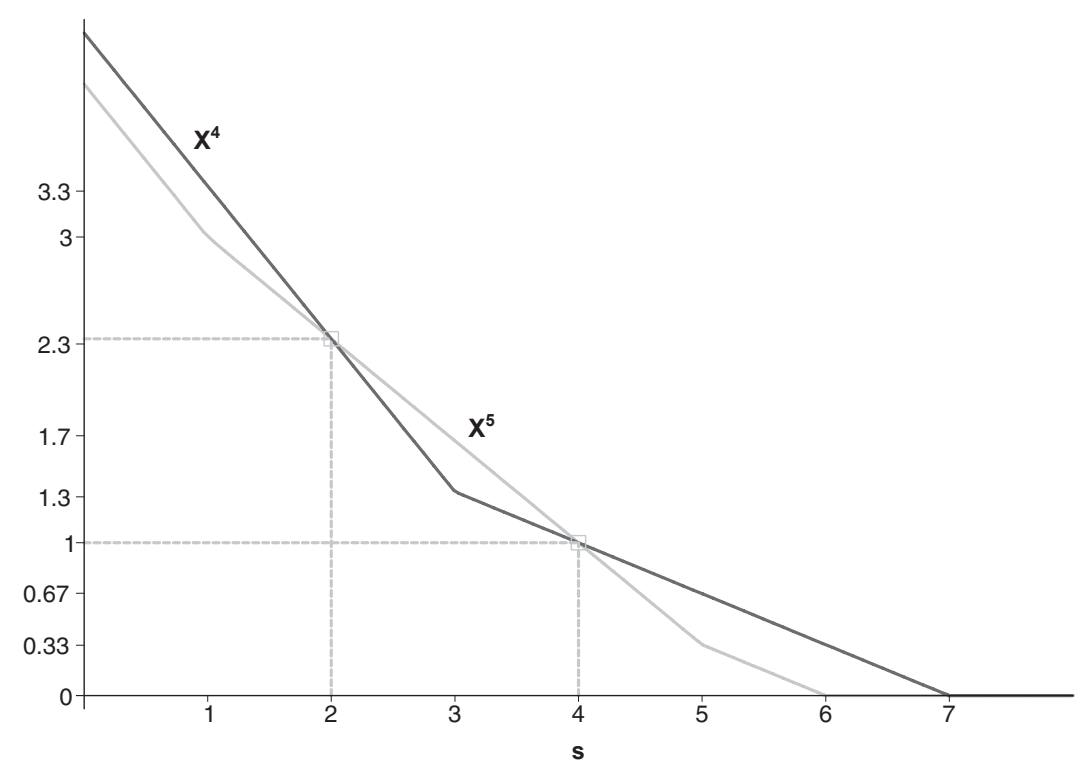

more efficiently and less equally distributed individual endowments. Inequality reduction is commonly associated in welfare economics with the Lorenz criterion or equivalently with the 
concept of majorisation (see Marshall and Olkin (1979)). More precisely, given two profiles $\mathbf{x}, \mathbf{y} \in \mathscr{S}(D)$ with $n(\mathbf{x})=n(\mathbf{y})=n$, we will say that profile $\mathbf{x}$ Lorenz dominates profile $\mathbf{y}$, which we write $\mathbf{x} \geq_{L} \mathbf{y}$, if:

$$
\begin{aligned}
& \int_{0}^{p} Q(\xi ; \mathbf{x}) d \xi \geqq \int_{0}^{p} Q(\xi ; \mathbf{y}) d \xi, \forall p \in[0,1), \text { and } \\
& \mu(\mathbf{x}) \equiv \int_{0}^{1} Q(\xi ; \mathbf{x}) d \xi=\int_{0}^{1} Q(\xi ; \mathbf{y}) d \xi \equiv \mu(\mathbf{y}) .
\end{aligned}
$$

By definition, Lorenz domination of one distribution by another assumes that the distributions have equal mean. As it is well-known, if distribution $\mathbf{x}$ is ranked above distribution $\mathbf{y}$ by the Lorenz quasi-ordering, then $\mathbf{y}$ obtains from $\mathbf{x}$ by means of a finite sequence of regressive transfers, and conversely. Then, we have the following remark that builds upon Marshall and Olkin (1979, 5A9).

Figure 3.6: Reverse generalised Lorenz dominating and dominated profiles

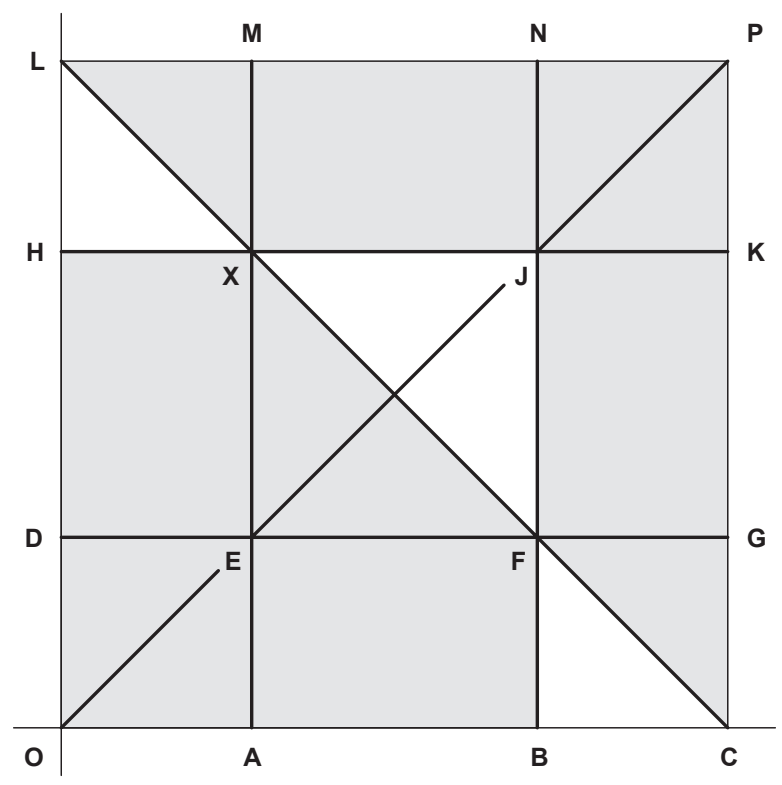

REMARK 3.1. Let $\mathbf{x}, \mathbf{y} \in \mathscr{S}(D)$ with $n(\mathbf{x})=n(\mathbf{y})$ and $\mu(\mathbf{x}) \geqq \mu(\mathbf{y})$. Then, we have:

$$
\mathbf{x} \geq_{\mathscr{V}_{2}} \mathbf{y} \Longleftrightarrow \exists \mathbf{z} \in \mathscr{S}(D) \mid \mathbf{z} \geq_{L} \mathbf{x} \text { and } \mathbf{z} \geq \mathbf{y}
$$

This clarifies the way efficiency and inequality contribute to the ranking of profiles when unanimity is required to hold among all those making judgements and whose value functions are non-decreasing and convex. ${ }^{4}$ Incidently, it is a direct consequence of Remark 3.1 that, if two profiles are ranked by the reverse generalised Lorenz criterion, then the dominating profile can be obtained from the dominated one by means of a finite sequence of increments

${ }^{4}$ This has to be contrasted with standard second degree stochastic dominance where efficiency and equality improvements result in higher ranked profiles. 
and/or regressive transfers. The difference between the generalised Lorenz and the reverse generalised Lorenz criteria is best illustrated by means of a simple figure when there are only two individuals. To this aim consider the profile $\mathbf{x}^{6}=(2,5)$ identified with point $\mathrm{X}$ in Figure 3.6. The shaded areas represent the sets of profiles that are dominated by and that dominate $\mathbf{x}^{6}$ according to the reverse generalised quasi-ordering. The profile $\mathbf{x}^{6}$ is dominated by all the profiles located in the area LXJFCP, while it dominates all the profiles in the area HXFBO. The area MXKP represents the profiles that vector dominate $\mathbf{x}^{6}$, while the area NFGP represents those profiles that vector dominate the permutation of $\mathbf{x}^{6}$ (point F). Therefore MXJFGP $=$ MXKP $\cup$ NFGP consists of the profiles that are ranked above $\mathbf{x}^{6}$ by the reverse quantile quasi-ordering. All the points in the area LXM can be obtained by means of an increment from a point located on the segment LX, which in turn results from profile $\mathbf{x}^{6}$ by a regressive transfer. It follows that all the profiles in the area LXM are considered no worse than $\mathbf{x}^{6}$ by the reverse generalised Lorenz quasi-ordering. A similar reasoning applies to the set FGC as compared to the permutation of $\mathbf{x}^{6}$ indicated by point $\mathrm{F}$. Since reverse quantile dominance implies reverse generalised Lorenz dominance, we conclude that LXJFCP $=$ LXM $\cup$ MXJFGP $\cup$ FGC. One would similar prove that HXFBO represents the set of profiles that are reversed generalised Lorenz dominated by $\mathbf{x}^{6}$. For memory, all the profiles in the area MXFGP generalised Lorenz dominate the profile $\mathbf{x}^{6}$, which in turn generalised Lorenz dominates all the profiles located in the area LXEFCO.

The convexity of the value function guarantees that a regressive transfer will always result in an increase in performance. Now, we will take the argument one step further and claim that the magnitude of the impact on performance of a regressive transfer depends on its location on the attribute scale. It is not unreasonable to posit that a regressive transfer that takes place among more endowed individuals has more impact on performance than a regressive transfer of the same magnitude but taking place among less endowed individuals. This idea is reminiscent of the notion of a diminishing progressive transfer introduced by Kolm (1976) and subsequently generalised by Shorrocks and Foster (1987). Given two profiles $\mathbf{x}, \mathbf{y} \in \mathscr{S}(D)$ with $n(\mathbf{x})=n(\mathbf{y})$, we will say that profile $\mathbf{x}$ is obtained from profile $\mathbf{y}$ by means of a augmenting regressive transfer if there exists $\Delta>0$ and four individuals $i, j, h$ and $k$ with $i<j<k$ and $i<h<k$ such that

$$
\begin{aligned}
& x_{g}=y_{g}, \forall h \neq i, j, h, k ; \\
& y_{i}-x_{i}=y_{j}-x_{j}=\Delta ; \quad x_{i} \leq x_{j} ; \\
& y_{h}-x_{h}=x_{k}-y_{k}=\Delta ; \quad y_{h} \leq y_{k} \\
& y_{j}-x_{i}=x_{k}-y_{h}=\xi ; \quad x_{i}<y_{h} .
\end{aligned}
$$

An augmenting regressive transfer combines a progressive transfer in the lower part of the distribution of endowments and a regressive transfer in the upper part of the distribution with the additional requirements that the mean and the variance are not affected. While it is immediately clear that the mean is not modified by an augmenting regressive transfer, it is also the case that the variance is unchanged as well. Consider any two profiles $\mathbf{x}$ and $\mathbf{y}$ such that condition (3.10) holds. Then we have

$$
\begin{aligned}
n\left[\sigma^{2}(\mathbf{x})-\sigma^{2}(\mathbf{y})\right] & =\left(x_{i}^{2}-y_{i}^{2}\right)+\left(x_{j}^{2}-y_{j}^{2}\right)+\left(x_{h}^{2}-y_{h}^{2}\right)+\left(x_{k}^{2}-y_{k}^{2}\right) \\
& =2 \Delta\left[\left(y_{k}-y_{h}\right)-\left(y_{j}-y_{i}\right)\right]+4 \Delta^{2}=0,
\end{aligned}
$$

since $y_{j}-y_{i}=y_{k}-y_{h}+2 \Delta$. Suppose we agree that the overall impact on performance of 
such a transformation is positive in which case we must have $W_{v}(\mathbf{x}) \geqq W_{v}(\mathbf{y})$ or equivalently:

$$
\left(v\left(y_{i}\right)-v\left(x_{i}\right)\right)-\left(v\left(x_{j}\right)-v\left(y_{j}\right)\right) \leqq\left(v\left(x_{h}\right)-v\left(y_{h}\right)\right)-\left(v\left(y_{k}\right)-v\left(x_{k}\right)\right) .
$$

Upon manipulating and integrating, condition (3.12) reduces to

$$
\int_{0}^{\Delta} \int_{0}^{\xi} v^{\prime \prime}\left(x_{i}+t+s\right) d t d s \leqq \int_{0}^{\Delta} \int_{0}^{\xi} v^{\prime \prime}\left(y_{h}+t+s\right) d t d s
$$

which, since $x_{i}<y_{h}$, holds if $v^{\prime \prime}(s)$ is non-decreasing with $s$. One can easily check that the latter condition is also necessary for performance to increase as the result of an augmenting regressive transfer. Suppose that $v^{\prime}$ is not convex, in which case there exist $s, t \in D$ with $s<t$ such that $2 v^{\prime}((s+t) / 2)>v^{\prime}(s)+v^{\prime}(t)$. Choose $\mathbf{x}=(s+\epsilon,(s+t) / 2,(s+t) / 2, t+\epsilon)$ and $\mathbf{y}=(s,((s+t) / 2)+\epsilon,((s+t) / 2)+\epsilon, t)$, so that $\mathbf{x}$ is obtained from $\mathbf{y}$ by means of a single augmenting regressive transfer. Then, for $\epsilon>0$ arbitrary small, we obtain

$$
v(s)+2 v\left(\frac{s+t}{2}+\epsilon\right)+v(t)>v(s+\epsilon)+2 v\left(\frac{s+t}{2}\right)+v(t+\epsilon),
$$

hence $W_{v}(\mathbf{y})>W_{v}(\mathbf{x}) .{ }^{5}$ For later reference we indicate by

$$
\mathscr{V}_{3}(D):=\left\{v \in \mathscr{V}(D) \mid v^{\prime}(s) \geqq 0, v^{\prime \prime}(s) \geqq 0 \text { and } v^{\prime \prime \prime}(s) \geqq 0, \forall s\right\}
$$

the class of non-decreasing, convex with increasing second derivatives for value functions. Since $\mathscr{V}_{3}(D)$ is a proper subset of $\mathscr{V}_{2}(D)$, the application of unanimity over the former class of value functions will result in a finer ranking than the one we get by imposing general agreement among the latter class. The following result identifies the procedure implementing unanimous agreement for all value functions in the class $\mathscr{V}_{3}(D)$.

Theorem 3.3. Let $\mathbf{x}, \mathbf{y} \in \mathscr{S}(D)$. Statements (a) and (b) below are equivalent:

(a) $W_{v}(\mathbf{x}) \geqq W_{v}(\mathbf{y})$, for all $v \in \mathscr{V}_{3}(D)$.

(b1) $\mu(\mathbf{x}) \geqq \mu(\mathbf{y})$ and

(b2) $\int_{s}^{\bar{s}} \int_{t}^{\bar{s}} D F(u ; \mathbf{x}) d u d t \geqq \int_{s}^{\bar{s}} \int_{t}^{\bar{s}} D F(u ; \mathbf{y}) d u d t$, for all $s \in[\underline{s}, \bar{s}]$.

When statements (b1) and (b2) of Theorem 3.3 hold, we will say profile $\mathbf{x}$ third order decumulative stochastic dominates profile $\mathbf{y}$. We first note that, contrary to what is the case in Theorems 3.1 and 3.2, there exists no procedure based on the reverse quantile function that permits to implement the unanimity of value judgements over the class $\mathscr{V}_{3}(D)$. This is a well-known result in the stochastic dominance literature, where the distribution function and quantile function approaches only coincide for the first and second orders. ${ }^{6}$ Integrating twice from $s$ to $\bar{s}$ the decumulative distribution functions and comparing the values obtained for all $s$ provide a practical means for checking if one profile is unanimously preferred to another when attention is restricted to value functions whose first derivative is positive, non-decreasing and

${ }^{5}$ Actually one only needs $n=3$ in order to prove that $v^{\prime}$ convex is necessary for performance to increase as the result of an augmenting regressive transfer (see e.g. Moyes and Shorrocks (1994))

6 The dominance criteria based on the quantile functions are related to the dual model of choice under risk introduced by Yaari (1987) and exploited later in the inequality literature by Ebert (1988), Yaari (1988) and more recently by Chateauneuf and Moyes (2004, 2006) and Magdalou and Moyes (2009). 
Figure 3.7: Double integrals of the decumulative distribution functions of $\mathbf{x}^{7}$ and $\mathbf{x}^{8}$

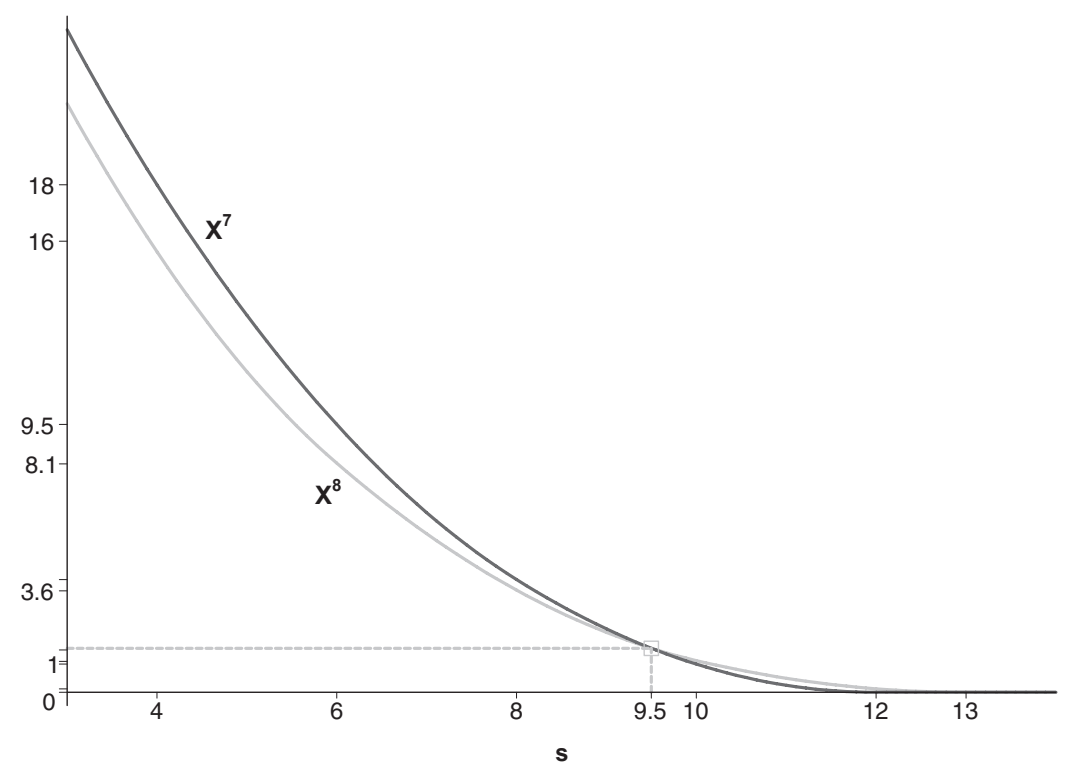

convex. Here again we would like to emphasise the distinction between standard cumulative stochastic dominance and the notion of dominance used in the paper. Standard second degree stochastic dominance requires that the cumulative distribution functions are integrated from $\underline{s}$ up to $s$. In our case, as in second order decumulative stochastic dominance, the double integrals are evaluated from $t$ and $s$ up to the upper bound $\bar{s}$. In general third order decumulative stochastic dominance and third order standard stochastic dominance lead to different rankings of profiles in terms of performance. The only case where the two procedures generates the same rankings is when the profiles under comparison have equal means and variance (see Section 6, Proposition 6.2). Finally, we note that it is necessary for profile $\mathbf{x}$ to third degree decumulative stochastic dominate profile $\mathbf{y}$ that

$$
(\mu(\mathbf{x})-\underline{s})^{2}+\sigma^{2}(\mathbf{x}) \geqq(\mu(\mathbf{y})-\underline{s})^{2}+\sigma^{2}(\mathbf{y})
$$

(see Section 6). In particular, this implies that, if the profiles $\mathbf{x}$ and $\mathbf{y}$ have the same mean, then the variance of $\mathbf{x}$ is no smaller than the variance of $\mathbf{y}$. That this condition is not sufficient is easily established by considering the profiles $\mathbf{x}^{7}=(4,8,12,12)$ and $\mathbf{x}^{8}=(6,6,10,13)$. Then, we have $\mu\left(\mathbf{x}^{7}\right)=9>8.75=\mu\left(\mathbf{x}^{8}\right)$ and $\sigma^{2}\left(\mathbf{x}^{7}\right)=11>8.69=\sigma^{2}\left(\mathbf{x}^{8}\right)$, hence condition (3.16) holds. However, the double integrals of the decumulative distribution functions of $\mathbf{x}^{7}$ and $\mathbf{x}^{8}$ cross as Figure 3.7 indicates and $\mathbf{x}^{7}$ does not dominate $\mathbf{x}^{8}$.

Admittedly, Theorem 3.3 is silent about the transformations of the profiles that underlie second degree decumulative stochastic dominance. That increments, regressive transfers and augmenting regressive transfers imply second degree decumulative stochastic domination of one profile over another follows directly from the definitions and Theorem 3.3. That the converse implication holds - if one profile second degree decumulative stochastically dominates another, then it can be obtained from the latter by means of a finite sequence of increments, regressive transfers and/or augmenting regressive transfers - follows from Shorrocks and Foster (1987). 


\section{Two Illustrations of the Measurement of Elitism}

\subsection{Affluence Versus Poverty}

In order to contrast affluence and poverty we have applied the generalised Lorenz and the reverse generalised Lorenz criteria to the comparison of 17 countries using income data from the Luxembourg Income Study (LIS). For each household, the LIS database indicates the disposable household income (DHI), that is its total income after taxation and transfer payments, and the household type determined by its composition and size. Incomes are provided in local currencies in order to facilitate comparisons within a country over time. These figures have been converted using the purchasing power parities (PPP) proposed by the OECD in order to make them comparable across countries. To take family needs into account it is necessary to adjust household incomes for size and for each household in the sample we have computed its equivalent income. Assuming independence of base level (see Blundell and Lewbel (1991)), the equivalent income of a household with income $y$ and size $m$ is given by $E(y ; m)=y / K(m)$, for all $m \geq 1$ and all $y \in D$, where $K(m)$ is the (relative) equivalence scale. Following Atkinson, Rainwater, and Smeeding (1995), we have chosen the isoelastic equivalence scale $K(m)=m^{\rho}$ with $\rho=.5 .{ }^{7}$ Table 4.1 gives the list of countries we have retained and indicates for each of

Table 4.1: The countries under comparison

\begin{tabular}{rlrcrrrr}
\hline No & Country & Year & Sample Size & Mean DHI & Std Dev & Minimum & Maximum \\
\hline 1 & Hungary & 1999 & 1927 & 6434.90 & 4262.95 & 1303.17 & 21928.93 \\
2 & Mexico & 2000 & 10072 & 6455.17 & 8311.57 & 360.83 & 39031.63 \\
3 & Poland & 1999 & 30812 & 7157.41 & 4432.45 & 1474.71 & 22828.70 \\
4 & Greece & 2000 & 3873 & 14759.51 & 10045.33 & 2063.03 & 48390.47 \\
5 & Netherlands & 1999 & 4331 & 19100.21 & 9124.06 & 1381.17 & 47538.52 \\
6 & Spain & 2000 & 4761 & 18972.04 & 13570.97 & 2244.69 & 66521.03 \\
7 & Germany & 2000 & 10982 & 20452.42 & 10401.80 & 3771.69 & 53899.97 \\
8 & Austria & 2000 & 2329 & 22146.59 & 11396.56 & 3706.64 & 60142.81 \\
9 & Sweden & 2000 & 14471 & 18984.00 & 9087.55 & 3485.15 & 48608.26 \\
10 & Canada & 2000 & 28902 & 24702.29 & 14499.66 & 3800.81 & 72485.77 \\
11 & Luxembourg & 2000 & 2415 & 30746.20 & 15704.40 & 10017.84 & 80973.26 \\
12 & Switzerland & 2000 & 3627 & 26803.70 & 14695.83 & 4805.17 & 76118.33 \\
13 & USA & 2000 & 49294 & 31638.14 & 26909.75 & 2640.00 & 132770.50 \\
14 & United Kingdom & 1999 & 24830 & 22169.08 & 15655.46 & 3886.12 & 76609.44 \\
15 & Belgium & 2000 & 2080 & 21266.58 & 11638.94 & 5010.52 & 60948.45 \\
16 & Norway & 2000 & 12870 & 25190.53 & 12745.74 & 4965.74 & 70526.72 \\
17 & Finland & 2000 & 10419 & 18816.83 & 9394.95 & 5527.89 & 51034.15 \\
\hline
\end{tabular}

these the year when the data were collected, the number of households in the sample, the mean of the corresponding adjusted DHIs, the standard deviation, and the minimum and maximum incomes.

The approaches in terms of poverty and affluence coincide at the first degree since they both imply that the value function is non-decreasing. This is no longer true for second and third degree dominance where affluence and poverty impose that the value function satisfies conflicting conditions. Concerning the second degree, we know from the definitions that, if

\footnotetext{
${ }^{7}$ While isoelastic equivalence scales are very specific and impose strong restrictions on the household's behaviour and on its members' preferences as shown by Ebert and Moyes (2011), they nevertheless provide rather good approximations of the scales currently used in empirical work (see Buhmann, Rainwater, Schmaus, and Smeeding (1988)).
} 
one country is ranked above another by the quantile quasi-ordering, then so it is according to the generalised Lorenz and the reverse generalised Lorenz quasi-orderings. Therefore, for a comparison of generalised Lorenz and reverse generalised Lorenz dominances to be meaningful, we have to restrict attention to those pairs of countries that are not ranked by the quantile criterion. Application of the generalised Lorenz quasi-ordering and of the reverse generalised Lorenz quasi-ordering to these 42 pairs of countries gives the results summarised in Table 4.2. We first note that together the generalised Lorenz and the reverse generalised Lorenz criteria allow to rank 38 pairs of countries out of the 42 for which the quantile criterion is not decisive. More important for our purpose is the fact that the generalised Lorenz and the reverse generalised Lorenz criteria give opposite conclusions with only one exception - Germany and Belgium - where they both agree. The reverse generalised Lorenz criterion appears to be more decisive than the generalised Lorenz criterion as 26 pairs of countries can be ranked by the former while the latter gives conclusive verdicts in only 12 cases. As the reverse generalised

Figure 4.1: Reverse quantile curves of Belgium and Germany

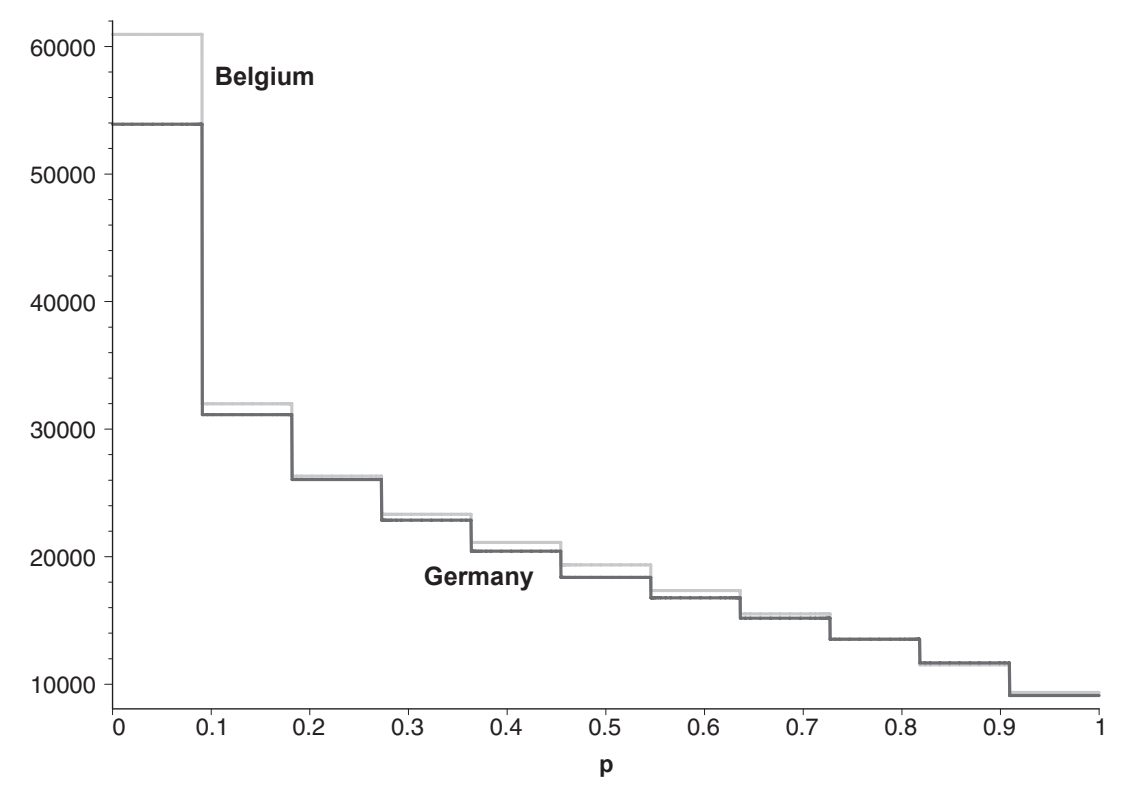

Lorenz dominance criterion favours both efficiency - a larger mean income is better - and inequality - a more unequal sharing of national income is better - the fact that it is more discriminatory for ranking countries is symptomatic of the fact that countries with larger per capita income tend to be also those where it is more unequally shared. To sum up, poverty and affluence do not appear to be two sides of the same coin - more affluence is not equivalent to less poverty - but are rather distinct features of the distributions. This is reflected in the definition of the criteria where partial sums are computed starting from low incomes to higher incomes in the case of the generalised Lorenz quasi-ordering whereas for the reverse generalised Lorenz quasi-ordering it is the contrary. The poorest individuals play a crucial role for generalised Lorenz dominance as a distribution cannot be ranked above another one if they have less income in the former distribution than in the latter. Similarly, for dominance in terms of reverse generalised Lorenz dominance it is necessary that the richest individuals do not experience a loss of income. The one case where both criteria agree is particularly interesting 


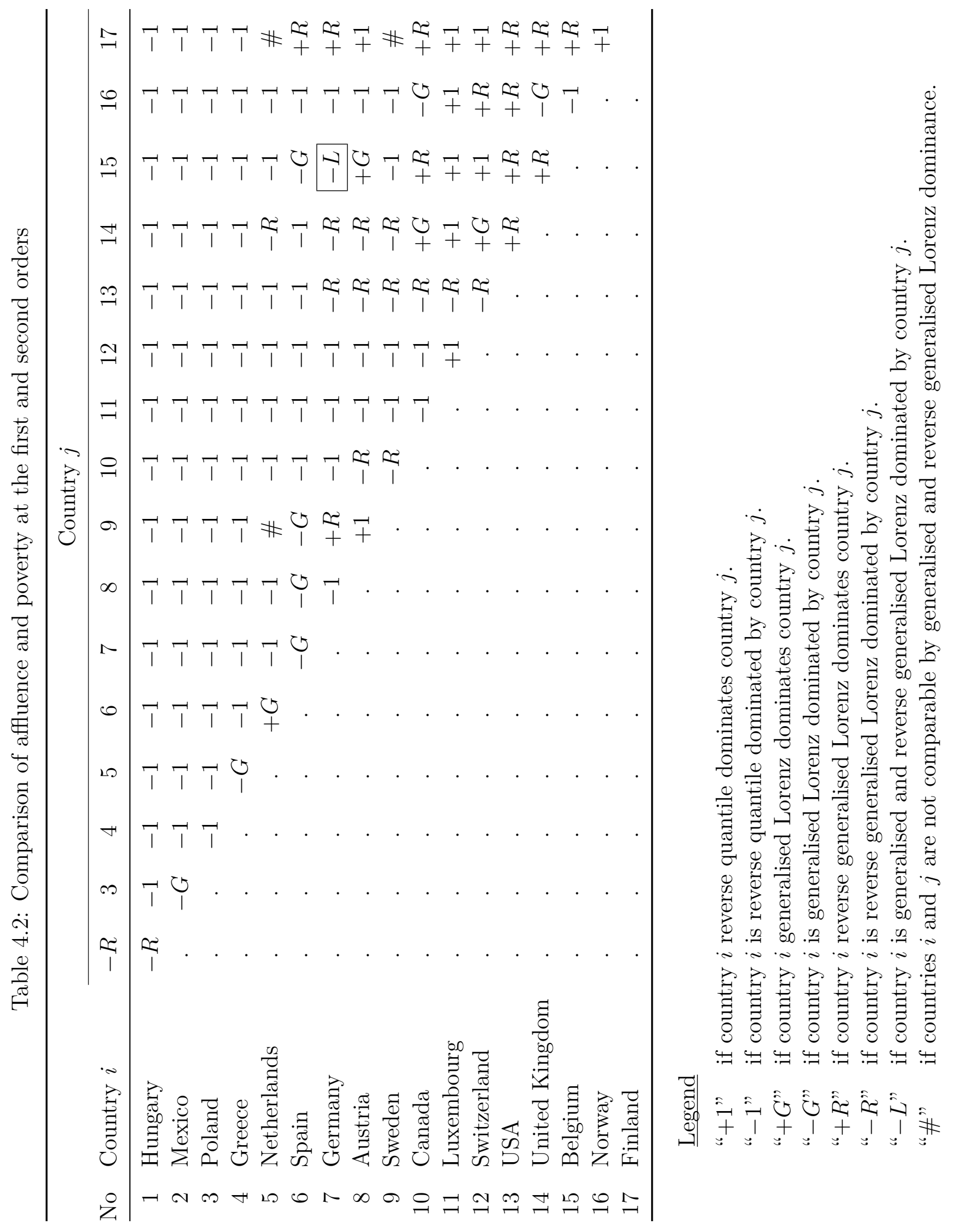


as it suggests that the dominating distribution is more polarised than the dominated one: there is relatively more affluence and less poverty in Belgium than in Germany. ${ }^{8}$ Inspection of Figure 4.1 reveals that the reverse quantile curve of Belgium intersects twice the reverse quantile curve of Germany first from above.

Three countries - the Netherlands, Sweden and Finland - cannot be compared by the generalised Lorenz and the reverse generalised Lorenz criteria. Inspection of Table 4.1 suggests that Finland might be ranked above Sweden which in turn might be ranked above the Netherlands when comparisons are made on the basis of third order - cumulative and decumulative stochastic dominance. Indeed, Finland has higher mean, minimal and maximal incomes than Sweden and the same is true for Sweden as compared to the Netherlands. However, for each of these three pairs of countries, either the double integrals of the cumulative distribution functions, or the double integrals of the survival functions, intersect at least once. Therefore, the recourse to the third order stochastic dominance tests does not help to resolve these cases of non-comparability.

\subsection{Scientific Performance}

In a second application, we use data derived from the Journal of Economic Literature (JEL) contents of journals for the period 1991 to 2000. These data were used in Lubrano et al. (2003) (see also Lubrano and Protopopescu (2004)) and were kindly provided by Michel Lubrano. For each publishing author in economics departments and economics research institutes in Europe, Lubrano et al. (2003) calculated an author score using the number of publications in five groups of journals. An article in a top journal is awarded five points and so on down to one point for the lowest quality journal referenced in the JEL. The scores recorded are for the ten year period 1991-2000. On the basis of these scores, Lubrano et al. (2003) construct an index to establish a ranking of economics departments, the first 20 of which are presented in Table 4.3. ${ }^{9}$ The first column indicates for each department its ranking in Lubrano et al. (2003)'s study while columns 3 to 6 provide some descriptive statistics. The London School of Economics is first followed by Tilburg and the Oxbridge departments. Within the countries covered, a disproportionate number of UK departments figure in the top 20 departments. We first note that the departments under consideration are of very unequal sizes ranging from around 300 affiliations for Cambridge, Oxford and the London School of Economics to less than 80 for Essex. The standard deviations of the three departments with the highest mean scores (Tilburg, Toulouse, Essex) are very different. Toulouse in particular has a high standard deviation which suggests that its high mean performance has probably to be imputed to a few academics. On the other hand, relatively low mean scores are generally associated with low values of the standard deviation (Bonn, Maastricht, Stockholm University, Warwick, Oxford, London Business School) which is an indication of a more equal distribution of the publication scores within the department.

\footnotetext{
${ }^{8}$ Such a situation is not exceptional as it can be checked by means of a simple example. Consider two income distributions $\mathbf{x}=(1.5,1.5,3.5)$ and $\mathbf{y}=(1,2,3)$ that are not comparable by means of the quantile criterion. One verifies that $\mathbf{x}$ dominates $\mathbf{y}$ according to both the generalised Lorenz and the reverse generalised Lorenz criteria. Actually, $\mathbf{x}$ can be obtained from $\mathbf{y}$ first by increasing the income of individual 1 by an amount 0.5 and then by transferring an amount 0.5 from individual 3 to individual 2. But $\mathbf{y}$ can also be transformed into $\mathrm{x}$ by means of an increment affecting individual 3 followed by a regressive transfer involving individuals 1 and 2.

${ }^{9}$ We were unable to reconstitute four of the departments ranked by Lubrano et al. (2003) which were aggregates of different research institutes.
} 
Table 4.3: The economic departments under comparison

\begin{tabular}{clrcrc}
\hline RANK & Department & AfFiliations & MEan & Std Dev & Maximum \\
\hline 1 & London School of Economics (LSE) & 334 & 12.77 & 21.80 & 180 \\
2 & Tilburg & 209 & 18.59 & 28.50 & 226 \\
3 & Oxford & 338 & 9.72 & 17.80 & 165 \\
4 & Cambridge & 290 & 10.74 & 18.90 & 227 \\
5 & Erasmus & 256 & 11.11 & 21.49 & 253 \\
6 & Louvain & 148 & 15.35 & 28.70 & 226 \\
7 & Amsterdam & 177 & 14.42 & 23.40 & 167 \\
8 & Warwick & 196 & 12.33 & 19.60 & 123 \\
9 & Toulouse & 115 & 18.85 & 43.30 & 267 \\
10 & University College London (UCL) & 176 & 17.73 & 31.10 & 212 \\
11 & Nottingham & 118 & 16.31 & 26.40 & 171 \\
12 & York & 132 & 12.40 & 18.70 & 94 \\
13 & Stockholm School of Economics (SSE) & 123 & 14.77 & 23.20 & 151 \\
14 & Maastricht & 145 & 12.78 & 19.40 & 120 \\
15 & Essex & 77 & 19.18 & 28.90 & 131 \\
16 & Universitat Autonoma Barcelona (UAB) & 113 & 13.34 & 20.50 & 105 \\
17 & Stockholm University (SU) & 106 & 11.97 & 22.10 & 154 \\
18 & Bonn & 96 & 12.32 & 18.80 & 132 \\
19 & London Business School (LBS) & 97 & 13.06 & 17.30 & 85 \\
20 & Free University Amsterdam (FUA) & 130 & 12.32 & 23.60 & 167 \\
\hline
\end{tabular}

Given the explicitly normative basis for ranking departments in terms of performance set out above, it will be interesting to see if a similar ranking is obtained when we apply our criteria. Since the latter are based on decumulative stochastic dominance, the number and direction of potential rankings can be reduced by looking first at the maximum score in a department. For example, since the maximum score is obtained by a member of the economics department at the University of Toulouse, this university cannot be ranked below any other in the list on the basis of the dominance criteria proposed above. Secondly, the average score of a department that is ranked above another by first order decumulative stochastic dominance cannot be lower. For instance, the fact that Louvain has a lower mean score than University College London prevents Louvain from being ranked above University College of London by first order stochastic dominance, even though the maximum score is higher for Louvain. Furthermore, the integral of the survival function over the range is equal to the mean score, so that a higher mean score is also a necessary condition for second order dominance as well. The economics department at the University of Essex has the highest mean score in our sample, and thus cannot be dominated by other departments. Therefore, Table 4.3 also defines the subset of potential dominance relations out of the 190 possible pairwise comparisons.

Application of first order and second order decumulative stochastic dominance to our 20 economics departments allows us to rank 89 pairs of departments among the 190 possible pairs which amounts to a success rate of about $47 \%$. Our results are summarised in Table 4.4 where the departments are listed according to the ranking of Lubrano et al. (2003). Surprisingly, first order decumulative stochastic dominance gives a conclusive verdict in 14 cases out of 190: for those pairs, whatever the threshold, the proportion of affiliated academics whose publication scores exceed this threshold value is greater in one department than in the other. From this point of view the cases of Tilburg, University College London, Nottingham and to a lesser extent Essex are worth noting: Tilburg dominates Oxford, Warwick, York, Maastricht and Universitat Autonoma Barcelona, University College London dominates the London School 


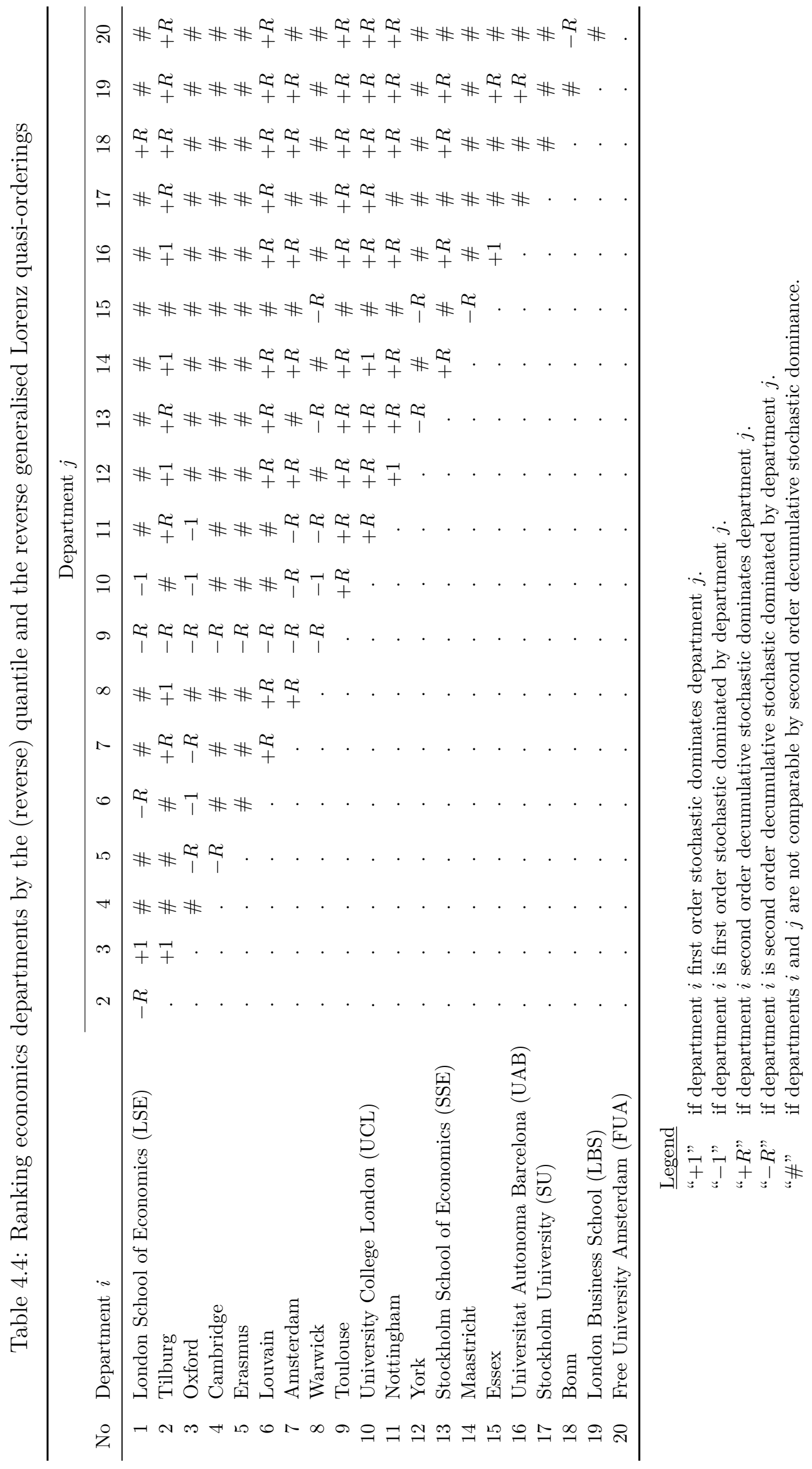


of Economics, Oxford, Warwick and Maastricht, Nottingham dominates Oxford and York, while Essex dominates Universitat Autonoma Barcelona, and none of these four departments appears to be dominated by another department at the first order.

Moving from first to second order decumulative stochastic dominance allows us to obtain conclusive verdicts in a further 75 cases (see Table 4.4). ${ }^{10}$ This is a relatively large number of rankings - nearly half of the maximum possible - for this type of approach (see for example the comparisons of income distributions above). Therefore, the introduction of the concern for elitism - captured by the convexity of the value function - enables a significant reduction in the number of inconclusive cases. The most important conclusion is that Toulouse dominates all the other departments with the exception of Essex. The reason why Toulouse does not rank above other departments at the first order is because the academics with the lowest scores in Toulouse do worse than the academics with the lowest scores in these departments. This is partly reflected by the fact that Toulouse has the largest variance in the publication scores. A second group of departments - dominated by Toulouse but not by Essex - are Tilburg, University College London and Louvain. On the basis of the transitive property of stochastic dominance relations, these three universities dominate the Free University of Amsterdam and Amsterdam, and the latter dominates the London School of Economics and the Stockholm School of Economics. Since the latter also dominates Warwick, York, Maastricht, Universitat Autonoma Barcelona, Bonn and the London Business School, so do all departments that dominate the Stockholm School of Economics (the three mentioned plus Amsterdam and Nottingham). It should be noted that it is not possible to rank Tilburg, the University College London and Louvain above Essex, Cambridge and Erasmus, and Louvain does not rank above Nottingham. The cases of Cambridge, Essex and Erasmus are worth mentioning: they cannot be ranked below the highest placed departments, but they do not rank above many lower-placed departments either. Erasmus only dominates Oxford and Cambridge, Essex only dominates Warwick and York, and Cambridge cannot be ranked above any department.

In order to verify how much at variance with the standard stochastic dominance approach the concept of elitism is, we have applied the generalised Lorenz criterion to our sample of economics departments (see Table A.1). We get a conclusive verdict for 72 pairs of departments out of the 176 pairs for which (reverse) quantile dominance is non-decisive. Contrary to what happened in our first illustration, application of generalised Lorenz dominance produces a score comparable with that obtained with the reverse generalised Lorenz dominance approach. A first consequence is that the application of generalised Lorenz dominance enables a number of indeterminacies obtained by the quantile criterion to be removed: both University College London and Nottingham dominate Cambridge, Erasmus and Louvain. Similarly, the Stockholm School of Economics and Maastricht dominate Oxford, Cambridge and Erasmus who are also dominated by Stockholm University, Bonn and the London Business School. Among the most interesting features that emerge as the result of the application of the standard generalised Lorenz criterion, we note that Essex, that was not comparable to Toulouse by reverse generalised Lorenz dominance, is now ranked above Toulouse by the generalised

\footnotetext{
${ }^{10}$ Admittedly, the attribute whose distribution is the object of comparisons is at best a very crude proxy of an academic's scientific performance. One way to acknowledge this imprecision would be to interpret an academic's publication score as an ordinal measure of her scientific performance, in which case moving from first degree stochastic dominance to second degree dominance will make little sense. Indeed, the resulting ranking of institutions would not be robust to increasing transformations of the individual performance scale. While we are aware of this difficulty, we nevertheless believe that this exercise might shed light on some important features of the academic landscape in Europe.
} 
Lorenz criterion. Actually, Essex is never dominated because it has the highest mean publication score, yet itself only dominates a few departments: in addition to dominating Toulouse by the standard generalised Lorenz criterion, Essex only dominates Warwick, the Stockholm School of Economics, Maastricht and the London Business School by the reversed generalised Lorenz criterion, and, as we already noted, Universitat Autonoma Barcelona by the quantile criterion. The cases of Tilburg and University College London are also worth noting: both dominate all the other departments - with the exceptions of University College London and Essex for Tilburg and Essex for University College London - by either the (reverse) quantile criterion, or the generalised Lorenz criterion, or the reverse generalised Lorenz criterion (see Table A.2). At the other extreme, the Oxford appears to be dominated at the second order by all departments with the exception of Warwick, York, Essex and Universitat Autonoma Barcelona. Things are less clearcut for Cambridge which is ranked above Oxford by the generalised Lorenz criterion and cannot be compared with Louvain, Warwick, York, Essex, Universitat Autonoma Barcelona and the Free University of Amsterdam at the second order. All together the generalised Lorenz and the reverse generalised Lorenz criteria lead to a conclusive verdict in 105 cases where the (reverse) quantile criterion was not decisive. The applications of generalised Lorenz and reverse generalised Lorenz dominance criteria provide contrasting pictures and suggest that economics departments have pursued different strategies. On the one hand, Toulouse has an elitist strategy with a relatively small group of top publishing academics but with also a fraction of the staff with considerably lower publication records. On the other hand, Essex has definitively a less elitist strategy and seems rather to look for a more homogenous staff in terms of publication scores.

It remains to be seen whether things improve when one applies third order decumulative stochastic dominance for comparing the economics departments. A look at Table 4.3 already makes clear that a number of departments will not pass the test. Consider for instance the 13 departments that second order decumulative stochastic dominance fails to compare with the London School of Economics. Clearly, Cambridge, Erasmus and York cannot be ranked above the London School of Economics by third order decumulative stochastic dominance since they have lower means and variances. Does it mean that Cambridge, Erasmus and York are dominated at the third order by the London School of Economics? Actually, the double integral of the decumulative distribution function of any of these departments intersects that of the London School of Economics. Condition (b2) of Theorem 3.3 is not fulfilled and it is therefore impossible to obtain a decisive verdict on the basis of third order decumulative stochastic dominance. Similarly, because the mean scores and the variances of Amsterdam, Nottingham, the Stockholm School of Economics and Essex are greater than those of the London School of Economics, it is possible for the former departments to dominate the latter. But here again comparison of the double integrals of the decumulative distribution functions of Amsterdam, Nottingham, the Stockholm School of Economics and Essex with that of the London School of Economics does not allow one arrive at conclusive verdicts. Actually similar conclusions hold for all pairs of departments that are not ranked by the second order decumulative stochastic dominance criterion and the application of third order decumulative stochastic dominance leads definitively to non-comparabilities.

\section{Concluding Remarks}

Up to now stochastic dominance has been used in comparisons of risky projects and of income distributions with emphasis placed on preferring distributions with more equality with par- 
ticular attention placed on what happens in the lower half of the distribution. The approach set out in this article suggests a further domain of application of stochastic dominance techniques. When elitism is important in a society or an institution, then performance rather than equality becomes the objective. Interest is concentrated more on what happens in the upper tail of the distribution. This position militates in favour of a convex utility function - or as we call it a value function - a condition whose implications for stochastic dominance had not been fully explored (see however Wong and Li (1999), Fishburn (1974) and Ross (1983)). ${ }^{11}$ The modified stochastic dominance criteria proposed here are useful for comparing distributions of incomes when affluence is society's objective and when comparing the scientific output of different research institutes or departments. The paper identifies the dominance tests to be implemented in order to decide whether one distribution is ranked above another by all utilitarian performance functions whose value functions are non-decreasing and convex. This demonstrates the flexibility and potential applicability of the stochastic dominance approach, that goes far beyond its traditional use in risk and welfare theories.

However, the approach expounded here is not without its limits. As a practical tool for making comparisons of distributions, the stochastic dominance approach produces a partial rather than a complete ranking of distributions. Part of the incompleteness of the ranking obtained is inherently linked to the necessary conditions for dominance such as the maximum income or publication score. This is the price paid for comparisons based on clearly specified value judgments rather than on an index which may be built on implicit and unstated normative criteria. However, nothing prevents us from considering the dominance tests introduced in this paper as a first round approach which may be supplemented in a second stage by the use of cardinal indices leading to a complete ordering of the profiles under comparison. For instance, using (2.5) and choosing specific forms for the value function would produce particular summary indices that could be used to resolve the incompleteness inherent in the dominance approach. In particular, letting $v(s)=s^{1-\eta} /(1-\eta)$ with $\eta \leqq 0$ would generate a family of indices analogous to the Atkinson-Kolm-Sen (AKS) family of evaluation functions. When $\eta=0$, we obtain $\Xi_{v}(\mathbf{x})=\mu(\mathbf{x})$, while $\Xi_{v}(\mathbf{x})=\max \left\{x_{i}\right\}$ when $\eta=-\infty$. Whatever the value of $\eta$, these indices will be monotone increasing: performance improves as the result of increments. For regressive transfers to increase performance as measured by such indices we have to restrict $\eta$ to be non-negative, while $\eta \leqq-1$ guarantees that augmenting regressive transfers have a positive impact on performance. ${ }^{12}$ The results extend to the generalised expected utility model of Quiggin (1993), that offers more flexibility than the standard expected utility model. This might be interesting as it allows the possibility to use different cardinal measures of performance like modifications of the Gini index.

A second weakness of our approach arises from the fact that the score variable aggregates different dimensions that all contribute to the performance of an institution. The evaluation of the research performance has given rise to widespread debates among the scientific community concerning in particular the appropriateness of the variables to be used. The choice of one variable among many others would not be that crucial if all the variables of potential interest appear to be correlated. However, there is evidence that the ranking of institutions varies

\footnotetext{
${ }^{11}$ To be more precise, it is the consequences of imposing increasingness and convexity on the utility function, that have not been investigated to the best of our knowledge. Notable exceptions are Makdissi and Wodon (2004) who applied criteria similar to ours to the measurement of the depletion rate of a natural resource, and Riese and Brunner (1998) who examine the severity of unemployment in terms of the spell lengths.

${ }^{12}$ These properties follow from Ebert (1988) who among other things provides a characterisation of the AKS family.
} 
with the choice of the variable retained for valuing publications. The evaluation of scientific performance involves at least two criteria that may not be correlated: quantity as measured for instance by the number of pages or articles published and quality approximated by the ranking of the journal where the article appeared. The latter can be determined by number of citations received by the journal or by its impact factors, or it can be derived from a general consensus among the profession. The way the scores are computed imposes arbitrary trade-offs between the different variables that are deemed relevant when assessing performance. Aggregating these dimensions into a single index as we did in Section 4.2 - where each academic is endowed with the number of articles he or she published multiplied by a variable measuring the quality of the journals - is not very informative. Indeed, two departments may rank closely even though they have adopted opposite publication strategies. For example, one department may have a high score based on the quantity of publications but these are concentrated in less prestigious journals, while another may have smaller quantitative output but concentrated in the top journals. A multidimensional approach building upon and extending the criteria devised in the inequality literature (see e.g. Atkinson and Bourguignon (1987), Bourguignon (1989), Bazen and Moyes (2003) or Gravel and Moyes (2008)) would overcome this drawback and seems more appropriate.

Finally, the normative nature of the stochastic dominance approach means that the axioms upon which the criteria for comparison are based may not be universally accepted: there are no right or wrong axioms. In particular, the relevance of the convexity of the value function, which captures the performance-improving nature of a regressive transfer, may be questioned. Clearly this form is relevant only in cases where it is accepted that society or an institution may benefit from greater inequality. An economic model which justifies the convexity of the value function may be useful in this context - for example, a model of the allocation of time between teaching and research in an economics department or a model of educational investment in a developing economy.

\section{Technicalities and Proofs}

The fact that we are working within a discrete framework imposes a particular structure that one should keep in mind throughout. In particular, it will be important in a later stage when the dominance criteria will be implemented. For the sake of interpretation, it may be helpful temporarily to conceive of the profile $\mathbf{x}:=\left(x_{1}, \ldots, x_{n(x)}\right)$ as an income distribution. Then we have

$$
\begin{aligned}
& F(s ; \mathbf{x})=\frac{q(s ; \mathbf{x})}{n(\mathbf{x})}=P_{1}(s ; \mathbf{x}) \text { and } \\
& 1-F(s ; \mathbf{x})=\frac{r(s ; \mathbf{x})}{n(\mathbf{x})}=R_{1}(s ; \mathbf{x})
\end{aligned}
$$

where by definition

$$
\begin{aligned}
& q(s ; \mathbf{x}):=\#\left\{i \in\{1,2, \ldots, n(\mathbf{x})\} \mid x_{i} \leqq s\right\} \text { and } \\
& r(s ; \mathbf{x}):=\#\left\{i \in\{1,2, \ldots, n(\mathbf{x})\} \mid x_{i}>s\right\}
\end{aligned}
$$

are respectively the number of poor and rich individuals in situation $\mathbf{x}$ when the poverty line is set equal to $s$. The quantity $P_{1}(s ; \mathbf{x})$ represents the percentage of individuals who in situation $\mathbf{x}$ are not above the poverty line $s$. Symmetrically, $R_{1}(s ; \mathbf{x})$ is the percentage of individuals 
in situation $\mathbf{x}$ above the poverty line $s$ and it may be interpreted as a measure of affluence in the society. Integrating $F(s ; \mathbf{x})$ and $1-F(s ; \mathbf{x})$, we obtain

$$
\begin{aligned}
& \int_{\underline{s}}^{s} F(t ; \mathbf{x}) d t=\frac{1}{n(\mathbf{x})} \sum_{i=1}^{q(s ; \mathbf{x})}\left(s-x_{i}\right)=P_{2}(s ; \mathbf{x}) \text { and } \\
& \int_{s}^{\bar{s}}(1-F(t ; \mathbf{x})) d t=\frac{1}{n(\mathbf{x})} \sum_{i=r(s ; \mathbf{x})}^{n(\mathbf{x})}\left(x_{i}-s\right)=R_{2}(s ; \mathbf{x}) .
\end{aligned}
$$

The quantity $P_{2}(s ; \mathbf{x})$ is the so-called average poverty gap, which represents the income needed in order to alleviate poverty deflated by the population size. Symmetrically, $R_{2}(s ; \mathbf{x})$ is the average income in excess to the poverty line, which we would be inclined to interpret as a measure of average affluence: throwing away this amount of income has no impact on poverty. Integrating again, we get

$$
\begin{aligned}
& \int_{\underline{s}}^{s} \int_{\underline{s}}^{t} F(u ; \mathbf{x}) d u d t=\frac{1}{n(\mathbf{x})^{2}} \sum_{i=1}^{q(s ; \mathbf{x})}\left(s-x_{i}\right)^{2}=P_{3}(s ; \mathbf{x}) \text { and } \\
& \int_{s}^{\bar{s}} \int_{t}^{\bar{s}}(1-F(u ; \mathbf{x})) d u d t=\frac{1}{n(\mathbf{x})^{2}} \sum_{i=r(s ; \mathbf{x})}^{n(\mathbf{x})}\left(x_{i}-s\right)^{2}=R_{3}(s ; \mathbf{x}) .
\end{aligned}
$$

To simplify the notation we let $F(s)=F(s ; \mathbf{x})$ and $G(s)=F(s ; \mathbf{y})$ represent the cdfs of distributions (or profiles) $\mathbf{x}$ and $\mathbf{y}$. The ddfs of $\mathbf{x}$ and $\mathbf{y}$ are therefore given by $1-F(s)$ and $1-G(s)$, respectively. Similarly, we indicate by $f(s)=f(s ; \mathbf{x})$ and $g(s)=g(s ; \mathbf{y})$ their density functions. Since by definition the utilitarian rule is invariant with respect to a replication - in other words it verifies the principle of population (see Dalton (1920)) - we assume without loss of generality that the profiles $\mathbf{x}$ and $\mathbf{y}$ have the same dimension: hence $n(\mathbf{x})=n(\mathbf{y})=n \geqq 2$.

Before we turn to the proofs of Theorems 3.1, 3.2 and 3.3 we find it useful to establish a number of facts.

MeAn. By definition of the mean, we have

$$
\mu(F)=\int_{\underline{s}}^{\bar{s}} s f(s) d s=\bar{s}-\int_{\underline{s}}^{\bar{s}} F(s) d s,
$$

from which we get

$$
\int_{\underline{s}}^{\bar{s}} F(s) d s=\bar{s}-\mu(F)
$$

Integrating $1-F(s)$ over $[\underline{s}, \bar{s}]$, we get:

$$
\int_{\underline{s}}^{\bar{s}}(1-F(s)) d s=(\bar{s}-\underline{s})-\int_{\underline{s}}^{\bar{s}} F(s) d s,
$$

which, upon using (6.6), reduces to

$$
\int_{\underline{s}}^{\bar{s}}(1-F(s)) d s=\mu(F)-\underline{s} .
$$

Combining (6.6) and (6.8), we obtain

$$
\mu(F)=\bar{s}-\int_{\underline{s}}^{\bar{s}} F(s) d s=\underline{s}+\int_{\underline{s}}^{\bar{s}}(1-F(s)) d s .
$$


Second Order Stochastic Dominances. Assume that $F$ is second degree stochastically dominated by $G$ so that

$$
\int_{\underline{s}}^{s}[F(t)-G(t)] d t \geqq 0, \forall s \in[s, \bar{s}]
$$

Suppose now that $\mu(F)=\mu(G)$, which, upon using (6.9), is equivalent to

$$
\int_{\underline{s}}^{\bar{s}}[F(s)-G(s)] d s=\int_{\underline{s}}^{\bar{s}}[(1-F(s))-(1-G(s))] d s=0 .
$$

This implies that

$$
\int_{\underline{s}}^{s}[(1-F(t))-(1-G(t))] d t+\int_{s}^{\bar{s}}[(1-F(t))-(1-G(t))] d t=0,
$$

which can be rewritten as

$$
\int_{\underline{s}}^{s}[G(t)-F(t)] d t+\int_{s}^{\bar{s}}[(1-F(t))-(1-G(t))] d t=0,
$$

and holds for all $s \in[s, \bar{s}]$. It follows from (6.10) that the first term of (6.13) is less or equal to zero and we therefore deduce that

$$
\int_{s}^{\bar{s}}[(1-F(t))-(1-G(t))] d t \geqq 0, \forall s \in[s, \bar{s}] .
$$

The argument works the other way round and we conclude (see also Shaked and Shanthikumar (1994, p. 56)):

Proposition 6.1. If $F$ and $G$ have equal means, then $F$ is second order stochastically dominated by $G$ in the usual sense if and only if $F$ second order stochastically dominates $G$ in the survival sense.

VARIANCE. By definition of the variance we have

$$
\sigma^{2}(F)=\bar{s}^{2}-\mu(F)^{2}-2 \int_{\underline{s}}^{\bar{s}} s F(s) d s .
$$

Integrating by parts the last integral in (6.15), we obtain

$$
\sigma^{2}(F)=\bar{s}^{2}-\mu(F)^{2}-2 \bar{s} \int_{\underline{s}}^{\bar{s}} F(s) d s+2 \int_{\underline{s}}^{\bar{s}} \int_{\underline{s}}^{s} F(t) d t d s .
$$

Using (6.6) and upon substitution into (6.16), we get

$$
\sigma^{2}(F)=-(\bar{s}-\mu(F))^{2}+2 \int_{\underline{s}}^{\bar{s}} \int_{\underline{s}}^{s} F(t) d t d s,
$$

from which we deduce that

$$
\int_{\underline{s}}^{\bar{s}} \int_{\underline{s}}^{s} F(t) d t d s=\frac{(\bar{s}-\mu(F))^{2}+\sigma^{2}(F)}{2} .
$$

Third Order Stochastic Dominances. Assume that $F$ is third degree stochastically dominated by $G$ so that

$$
\int_{\underline{s}}^{s} \int_{\underline{s}}^{t}[F(u)-G(u)] d u d t \geqq 0, \forall s \in[s, \bar{s}] .
$$


Suppose now that $\mu(F)=\mu(G)$ and $\sigma^{2}(F)=\sigma^{2}(G)$, which upon using (6.18) is equivalent to

$$
\int_{\underline{s}}^{\bar{s}} \int_{\underline{s}}^{t}[F(u)-G(u)] d u d t=\int_{\underline{s}}^{\bar{s}} \int_{\underline{s}}^{t}[(1-F(u))-(1-G(u))] d u d t=0 .
$$

This implies that

$$
\int_{\underline{s}}^{s} \int_{\underline{s}}^{t}[(1-F(u))-(1-G(u))] d u d t+\int_{s}^{\bar{s}} \int_{\underline{s}}^{t}[(1-F(u))-(1-G(u))] d u d t=0,
$$

which can be rewritten as

$$
\int_{\underline{s}}^{s} \int_{\underline{s}}^{t}[G(u)-F(u)] d u d t+\int_{s}^{\bar{s}} \int_{\underline{s}}^{t}[(1-F(u))-(1-G(u))] d u d t=0,
$$

and holds for all $s \in[s, \bar{s}]$. It follows from (6.19) that the first term of (6.22) is less or equal to zero and we therefore deduce that

$$
\int_{s}^{\bar{s}} \int_{\underline{s}}^{t}[(1-F(u))-(1-G(u))] d u d t \geqq 0,
$$

which holds true for all $s \in[\underline{s}, \bar{s}]$. The argument works the other way round and we conclude:

Proposition 6.2. If $F$ and $G$ have equal means and variances, then $F$ is third order stochastically dominated by $G$ in the usual sense if and only if $F$ third order stochastically dominates $G$ in the survival sense.

We are now well equipped and can proceed to the proofs of Theorems 3.1, 3.2 and 3.3 successively.

Theorem 3.1. It is obvious from the definitions that the ranking of profiles implied by the first order decumulative stochastic dominance criterion is identical to that generated by standard first order stochastic dominance. Similarly, the rankings of profiles resulting from the application of the quantile dominance criterion and the reverse quantile dominance criterion are identical. We therefore refer the reader to the standard proofs in the stochastic dominance literature (see e.g. Saposnik (1981), Fishburn and Vickson (1978)).

Theorem 3.2. We prove successively the following two chains of implications: $(c) \Longrightarrow(a) \Longrightarrow$ $(c)$ and $(b) \Longrightarrow(a) \Longrightarrow(b)$.

$(c) \Longrightarrow(a)$. We have to show that, if condition (b) holds, then

$$
W_{v}(\mathbf{x})-W_{v}(\mathbf{y})=\frac{1}{n} \sum_{i=1}^{n} \frac{v\left(x_{i}\right)-v\left(y_{i}\right)}{x_{i}-y_{i}}\left[x_{i}-y_{i}\right] \geqq 0,
$$

for all $v \in \mathscr{V}_{2}(D)$. Applying Abel's decomposition rule to (6.24), we obtain

$$
\begin{aligned}
W_{v}(\mathbf{x})-W_{v}(\mathbf{y}) & =\frac{v\left(x_{1}\right)-v\left(y_{1}\right)}{x_{1}-y_{1}} \frac{1}{n} \sum_{h=1}^{n}\left[x_{h}-y_{h}\right] \\
& +\sum_{i=2}^{n}\left(\frac{v\left(x_{i}\right)-v\left(y_{i}\right)}{x_{i}-y_{i}}-\frac{v\left(x_{i-1}\right)-v\left(x_{i-1}\right)}{x_{i-1}-y_{i-1}}\right) \frac{1}{n} \sum_{h=i}^{n}\left[x_{h}-y_{h}\right] \geqq 0 .
\end{aligned}
$$


The first fraction on the rhs of (6.25) is non-negative because $v$ is non-decreasing. It can be shown that the convexity of $v$ guarantees that the difference of fractions within large brackets on the rhs of (6.25) is also non-negative. We therefore conclude that

$$
\frac{1}{n} \sum_{h=i}^{n} x_{h} \geqq \frac{1}{n} \sum_{h=i}^{n} y_{h}, \forall i=1,2, \ldots, n,
$$

is a sufficient condition for (6.24) to hold.

$(a) \Longrightarrow(c)$. We argue a contrario and show that, if condition (c) does not hold, then nor does condition (a). Let $k^{*}$ be the smallest $k$ such that $x_{n}+x_{n-1}+\cdots+x_{k+1}+x_{k}<y_{n}+y_{n-1}+$ $\cdots+y_{k+1}+y_{k}$, which implies that $x_{k^{*}}<y_{k^{*}}$. Choosing

$$
v(s):= \begin{cases}0, & \text { for } s \leq x_{k^{*}} \\ s-x_{k^{*}}, & \text { for } x_{k^{*}}<s\end{cases}
$$

which is non-decreasing and convex, we obtain

$$
\sum_{i=1}^{n} v^{*}\left(y_{i}\right) \geqq y_{n}+\cdots+y_{k^{*}+1}+y_{k^{*}}>x_{n}+\cdots+x_{k^{*}+1}+x_{k^{*}}=\sum_{i=1}^{n} v^{*}\left(x_{i}\right) .
$$

The proof is made complete by noting that $v^{*}$ can be approximated as closely as desired by a differentiable non-decreasing and convex function.

$(b) \Longrightarrow(a)$. We have to show that, if condition (b) holds, then

$$
W_{v}(\mathbf{x})-W_{v}(\mathbf{y})=\int_{\underline{s}}^{\bar{s}} v(s)[f(s)-g(s)] d s \geqq 0,
$$

for all $v \in \mathscr{V}_{2}(D)$. Upon integrating by parts (6.29) twice and using the fact that $F(\bar{s})=$ $G(\bar{s})=1$, this reduces to

$$
-v^{\prime}(\bar{s}) \int_{\underline{s}}^{\bar{s}}[F(s)-G(s)] d s+\int_{\underline{s}}^{\bar{s}} v^{\prime \prime}(s) \int_{\underline{s}}^{s}[F(t)-G(t)] d t d s \geqq 0,
$$

which we can rewrite equivalently as

$$
v^{\prime}(\bar{s}) \int_{\underline{s}}^{\bar{s}}[(1-F(s))-(1-G(s))] d s-\int_{\underline{s}}^{\bar{s}} v^{\prime \prime}(s) \int_{\underline{s}}^{s}[(1-F(t))-(1-G(t))] d t d s \geqq 0 .
$$

Using (6.4), the fact that

$$
\begin{aligned}
\int_{\underline{s}}^{s}[(1-F(t)) & -(1-G(t))] d t=\int_{\underline{s}}^{\bar{s}}[(1-F(t))-(1-G(t))] d t \\
& -\int_{s}^{\bar{s}}[(1-F(t))-(1-G(t))] d t,
\end{aligned}
$$

for all $s \in[s, \bar{s}]$, and upon substituting into (6.31), we obtain

$$
v^{\prime}(\bar{s})[\mu(F)-\mu(G)]-\int_{\underline{s}}^{\bar{s}} v^{\prime \prime}(s)\left[\mu(F)-\mu(G)-\int_{s}^{\bar{s}}[(1-F(t))-(1-G(t))] d t\right] d s \geqq 0,
$$

or equivalently

$$
(\mu(F)-\mu(G))\left[v^{\prime}(\bar{s})-\int_{\underline{s}}^{\bar{s}} v^{\prime \prime}(s) d s\right]+\int_{s}^{\bar{s}}[(1-F(t))-(1-G(t))] d t d s \geqq 0,
$$


which finally simplifies to

$$
v^{\prime}(\underline{s})[\mu(F)-\mu(G)]+\int_{\underline{s}}^{\bar{s}} v^{\prime \prime}(s) \int_{s}^{\bar{s}}[(1-F(t))-(1-G(t))] d t d s \geqq 0 .
$$

Using (6.8) and upon substitution into the first term of (6.35), we finally obtain

$$
v^{\prime}(\underline{s}) \int_{\underline{s}}^{\bar{s}}[(1-F(t))-(1-G(t))] d t+\int_{\underline{s}}^{\bar{s}} v^{\prime \prime}(s) \int_{s}^{\bar{s}}[(1-F(t))-(1-G(t))] d t d s \geqq 0 .
$$

Since by assumption $v^{\prime}(\bar{s}) \geqq 0$ and $v^{\prime \prime}(\bar{s}) \geqq 0$, for all $s \in(\underline{s}, \bar{s})$, we conclude that

$$
\int_{s}^{\bar{s}}(1-F(t)) d t \geqq \int_{s}^{\bar{s}}(1-G(t)) d t, \forall s \in[s, \bar{s}],
$$

is a sufficient condition for (6.36) - and thus statement (a) of Theorem 3.2 - to hold.

$(a) \Longrightarrow(b)$. Suppose condition (a) holds, which implies that it is verified in particular for all utility functions $v(t ; s):=\max \{0, s-t\}$. Then, we have

$$
\sum_{i=1}^{n} v\left(x_{i} ; s\right)=\sum_{i=r(s ; \mathbf{x})}^{n}\left(s-x_{i}\right) \geqq \sum_{i=r(s ; \mathbf{y})}^{n}\left(s-y_{i}\right)=\sum_{i=1}^{n} v\left(y_{i} ; s\right) .
$$

Since this is true for all $s \in[s, \bar{s}]$ and given (6.3b), we conclude that condition (b) holds.

Theorem 3.3. We prove successively that $(b) \Longrightarrow(a) \Longrightarrow(b)$.

$(b) \Longrightarrow(a)$. Integrating by parts $(6.35)$, we obtain

$$
\begin{aligned}
W_{v}(\mathbf{x})-W_{v}(\mathbf{y}) & =v^{\prime}(\underline{s})[\mu(F)-\mu(G)]+v^{\prime \prime}(\bar{s}) \int_{\underline{s}}^{\bar{s}} \int_{s}^{\bar{s}}[(1-F(t))-(1-G(t))] d t d s \\
& -\int_{\underline{s}}^{\bar{s}} v^{\prime \prime \prime}(s)\left[\int_{\underline{s}}^{s} \int_{t}^{\bar{s}}[(1-F(u))-(1-G(u))] d u d t\right] d s \geqq 0 .
\end{aligned}
$$

Noticing that

$$
\begin{aligned}
\int_{\underline{s}}^{s} \int_{t}^{\bar{s}}[(1-F(u)) & -(1-G(u))] d u d t=\int_{\underline{s}}^{\bar{s}} \int_{t}^{\bar{s}}[(1-F(u))-(1-G(u))] d u d t \\
& -\int_{s}^{\bar{s}} \int_{t}^{\bar{s}}[(1-F(u))-(1-G(u))] d u d t,
\end{aligned}
$$

for all $s \in[s, \bar{s}]$, and upon substituting into (6.39), we obtain

$$
\begin{aligned}
v^{\prime}(\underline{s})[\mu(F)-\mu(G)]+v^{\prime \prime}(\bar{s})\left[\int_{\underline{s}}^{\bar{s}} \int_{s}^{\bar{s}}[(1-F(t))-(1-G(t))] d t d s\right] \\
-\int_{\underline{s}}^{\bar{s}} v^{\prime \prime \prime}(s)\left[\int_{\underline{s}}^{\bar{s}} \int_{t}^{\bar{s}}[(1-F(u))-(1-G(u))] d u d t\right] d s \\
+\int_{\underline{s}}^{\bar{s}} v^{\prime \prime \prime}(s)\left[\int_{s}^{\bar{s}} \int_{t}^{\bar{s}}[(1-F(u))-(1-G(u))] d u d t\right] d s \geqq 0,
\end{aligned}
$$

or equivalently

$$
\begin{aligned}
& v^{\prime}(\underline{s})[\mu(F)-\mu(G)] \\
+ & {\left[\int_{\underline{s}}^{\bar{s}} \int_{s}^{\bar{s}}[(1-F(t))-(1-G(t))] d t d s\right]\left(v^{\prime \prime}(\bar{s})-\int_{\underline{s}}^{\bar{s}} v^{\prime \prime \prime}(s) d s\right) } \\
+ & \int_{\underline{s}}^{\bar{s}} v^{\prime \prime \prime}(s)\left[\int_{s}^{\bar{s}} \int_{t}^{\bar{s}}[(1-F(u))-(1-G(u))] d u d t\right] d s \geqq 0,
\end{aligned}
$$


which finally reduces to

$$
\begin{aligned}
& v^{\prime}(\underline{s})[\mu(F)-\mu(G)] \\
+ & v^{\prime \prime}(\underline{s})\left[\int_{\underline{s}}^{\bar{s}} \int_{s}^{\bar{s}}[(1-F(t))-(1-G(t))] d t d s\right] \\
+ & \int_{\underline{s}}^{\bar{s}} v^{\prime \prime \prime}(s)\left[\int_{s}^{\bar{s}} \int_{t}^{\bar{s}}[(1-F(u))-(1-G(u))] d u d t\right] d s \geqq 0 .
\end{aligned}
$$

Since by assumption $v^{\prime}(\bar{s}) \geqq 0, v^{\prime \prime}(\bar{s}) \geqq 0$ and $v^{\prime \prime \prime}(\bar{s}) \geqq 0$, for all $s \in(\underline{s}, \bar{s})$, we conclude that

$$
\begin{aligned}
& \mu(F) \geqq \mu(G) \text { and } \\
& \int_{s}^{\bar{s}} \int_{t}^{\bar{s}}(1-F(u)) d u d t \geqq \int_{s}^{\bar{s}} \int_{t}^{\bar{s}}(1-G(u)) d u d t, \forall s \in(\underline{s}, \bar{s}),
\end{aligned}
$$

are sufficient conditions for (6.43) - and thus statement (a) of Theorem 3.3 - to hold.

$(a) \Longrightarrow(b)$. Suppose condition (a) holds, which implies that it is verified in particular for all utility functions $v(t ; s):=\max \{0, s-t\}^{2}$. Then, we have

$$
\sum_{i=1}^{n} v\left(x_{i} ; s\right)=\sum_{i=r(s ; \mathbf{x})}^{n}\left(s-x_{i}\right)^{2} \geqq \sum_{i=r(s ; \mathbf{y})}^{n}\left(s-y_{i}\right)^{2}=\sum_{i=1}^{n} v\left(y_{i} ; s\right) .
$$

Since this is true for all $s \in[s, \bar{s}]$ and given (6.4b), we conclude that condition (b) holds.

Another Interesting EqUALITy. Integrating $1-F(s)$ over $[s, \bar{s}]$, we get:

$$
\int_{s}^{\bar{s}}(1-F(t)) d t=(\bar{s}-s)-\int_{s}^{\bar{s}} F(t) d t .
$$

Integrating (6.46) we obtain

$$
\int_{s}^{\bar{s}} \int_{t}^{\bar{s}}(1-F(u)) d u d t=\frac{1}{2}(\bar{s}-s)^{2}-\int_{s}^{\bar{s}} \int_{t}^{\bar{s}} F(u) d u d t,
$$

or equivalently

$$
\int_{s}^{\bar{s}} \int_{t}^{\bar{s}} F(u) d u d t=\frac{1}{2}(\bar{s}-s)^{2}-\int_{s}^{\bar{s}} \int_{t}^{\bar{s}}(1-F(u)) d u d t,
$$

which holds for all $s \in[\underline{s}, \bar{s}]$. In particular, setting $s=\underline{s}$, we get

$$
\int_{\underline{s}}^{\bar{s}} \int_{t}^{\bar{s}} F(u) d u d t=\frac{1}{2}(\bar{s}-\underline{s})^{2}-\int_{\underline{s}}^{\bar{s}} \int_{t}^{\bar{s}}(1-F(u)) d u d t .
$$

Upon manipulating the lhs of (6.49) and using (6.6), we obtain

$$
\begin{aligned}
\int_{\underline{s}}^{\bar{s}}\left[\int_{\underline{s}}^{\bar{s}} F(u) d u\right] d t & -\int_{\underline{s}}^{\bar{s}} \int_{\underline{s}}^{t} F(u) d u d t=\int_{\underline{s}}^{\bar{s}}[\bar{s}-\mu(F)] d s-\int_{\underline{s}}^{\bar{s}} \int_{\underline{s}}^{t} F(u) d u d t \\
& =(\bar{s}-\mu(F))(\bar{s}-\underline{s})-\int_{\underline{s}}^{\bar{s}} \int_{\underline{s}}^{t} F(u) d u d t .
\end{aligned}
$$

Substituting (6.50) into (6.49), we get

$$
(\bar{s}-\mu(F))(\bar{s}-\underline{s})-\int_{\underline{s}}^{\bar{s}} \int_{\underline{s}}^{t} F(u) d u d t=\frac{1}{2}(\bar{s}-\underline{s})^{2}-\int_{\underline{s}}^{\bar{s}} \int_{t}^{\bar{s}}(1-F(u)) d u d t,
$$


which, upon using (6.18), reduces to

$$
(\bar{s}-\mu(F))(\bar{s}-\underline{s})-\frac{1}{2}\left[(\bar{s}-\mu(F))^{2}+\sigma^{2}(F)\right]=\frac{1}{2}(\bar{s}-\underline{s})^{2}-\int_{\underline{s}}^{\bar{s}} \int_{t}^{\bar{s}}(1-F(u)) d u d t .
$$

All computation done, we finally get

$$
\int_{\underline{s}}^{\bar{s}} \int_{t}^{\bar{s}}(1-F(u)) d u d t=\frac{(\mu(F)-\underline{s})^{2}+\sigma^{2}(F)}{2},
$$

which is to be compared with (6.18). It is therefore necessary for condition (b2) of Theorem 3.3 to hold that

$$
(\mu(F)-\underline{s})^{2}+\sigma^{2}(F) \geqq(\mu(G)-\underline{s})^{2}+\sigma^{2}(G) .
$$

This implies in particular that, if the profiles $\mathbf{x}$ and $\mathbf{y}$ have the same mean - which rules out the possibility that one profile is obtained from the other by means of increments - then the variance of $\mathbf{x}$ cannot be smaller than the variance of $\mathbf{y}$. This has to be contrasted with standard third order stochastic dominance, which requires that the variance of the dominating situation be no larger than that of the dominated situation under the equal mean restriction.

\section{A. Scientific Performance: Additional Tables}

In order to get a more comprehensive picture of scientific performance in our sample of European economics departments, we contrast the rankings obtained on the basis of reverse generalised Lorenz dominance with that resulting from the application of the standard generalised Lorenz criterion. We have represented in Table A.1 the ranking of the European economics departments by the generalised Lorenz criterion or equivalently by standard second degree stochastic dominance. Table A.2 summarises the information provided by the application of reverse generalised Lorenz dominance and generalised Lorenz dominance. It makes clear that the rankings of the departments generated by these two criteria differ substantially, even though they coincide in a number of cases. Finally, Table A.3 permits to compare the discriminatory powers of the different dominance criteria when judging the scientific performance of the economics departments.

It is remarkable that the generalised Lorenz and reverse generalised Lorenz dominance criteria are equally discriminatory contrary to what happens for the comparison of countries on the basis of affluence and poverty. Among a total of 190 possible pairwise comparisons, the (reverse) quantile criterion gives a conclusive verdict for 14 pairs of departments which amounts to $7.36 \%$ of the total number of comparisons. Application of the reverse generalised Lorenz criterion allows us to obtain conclusive verdicts in a further 75 cases. In total, reverse generalised Lorenz dominance is conclusive in 89 cases, that is the 75 cases above plus the 14 cases ranked by the quantile criterion, hence $46.84 \%$ of the total number of comparisons. Similarly, the generalised Lorenz criterion allows us to obtain conclusive verdicts in 72 cases in addition to the 14 cases where the quantile criterion is decisive. Therefore, generalised Lorenz dominance is decisive in 86 cases (45.27\%) in a total of 190 possible cases. A total of 121 pairs of departments, for which the quantile criterion is non-decisive, can be ordered by either the reverse generalised Lorenz criterion, or the generalised Lorenz criterion. These two criteria agree on the ranking of departments in only 27 cases $(14.21 \%)$ out of the 176 where the quantile criterion cannot conclude. 


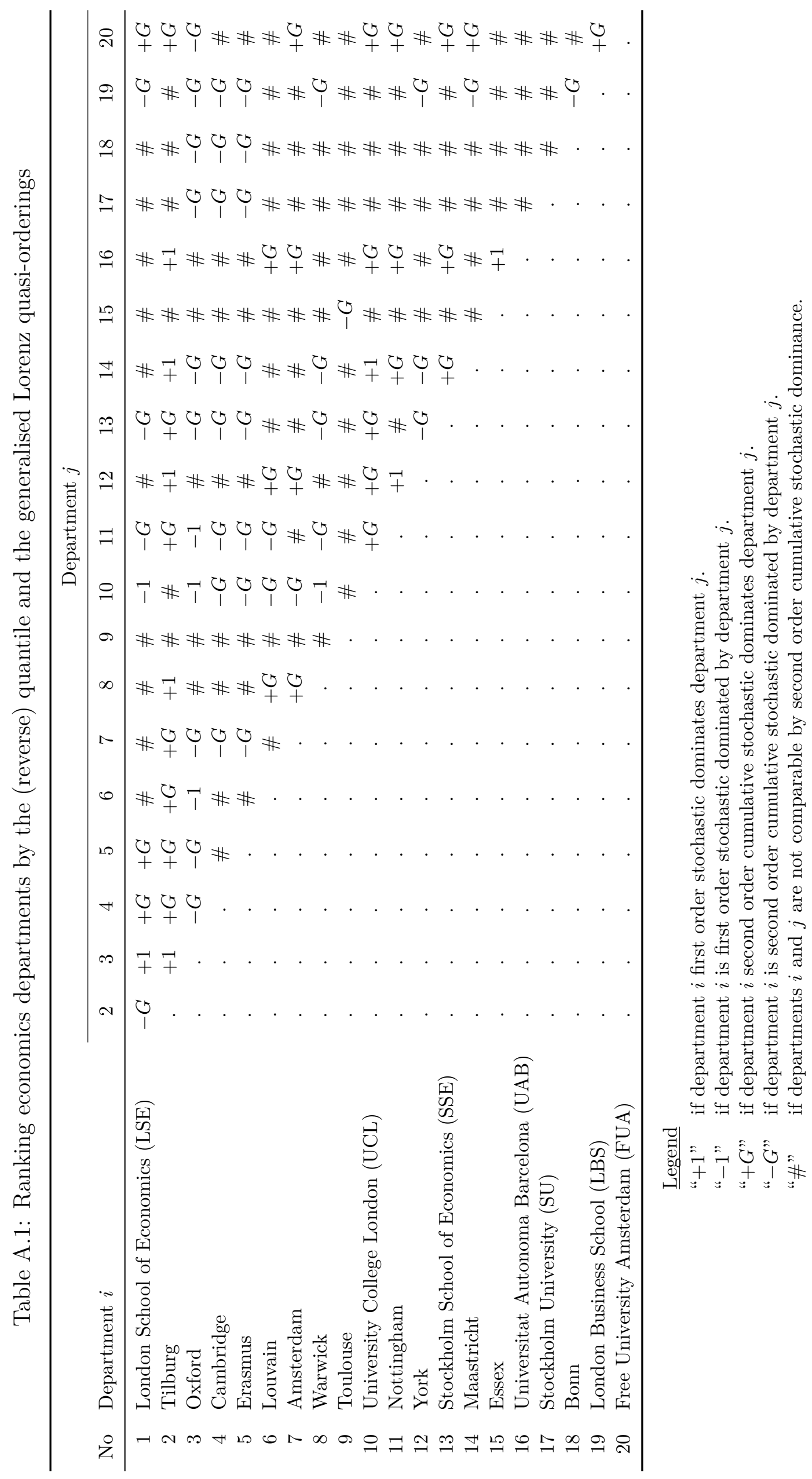




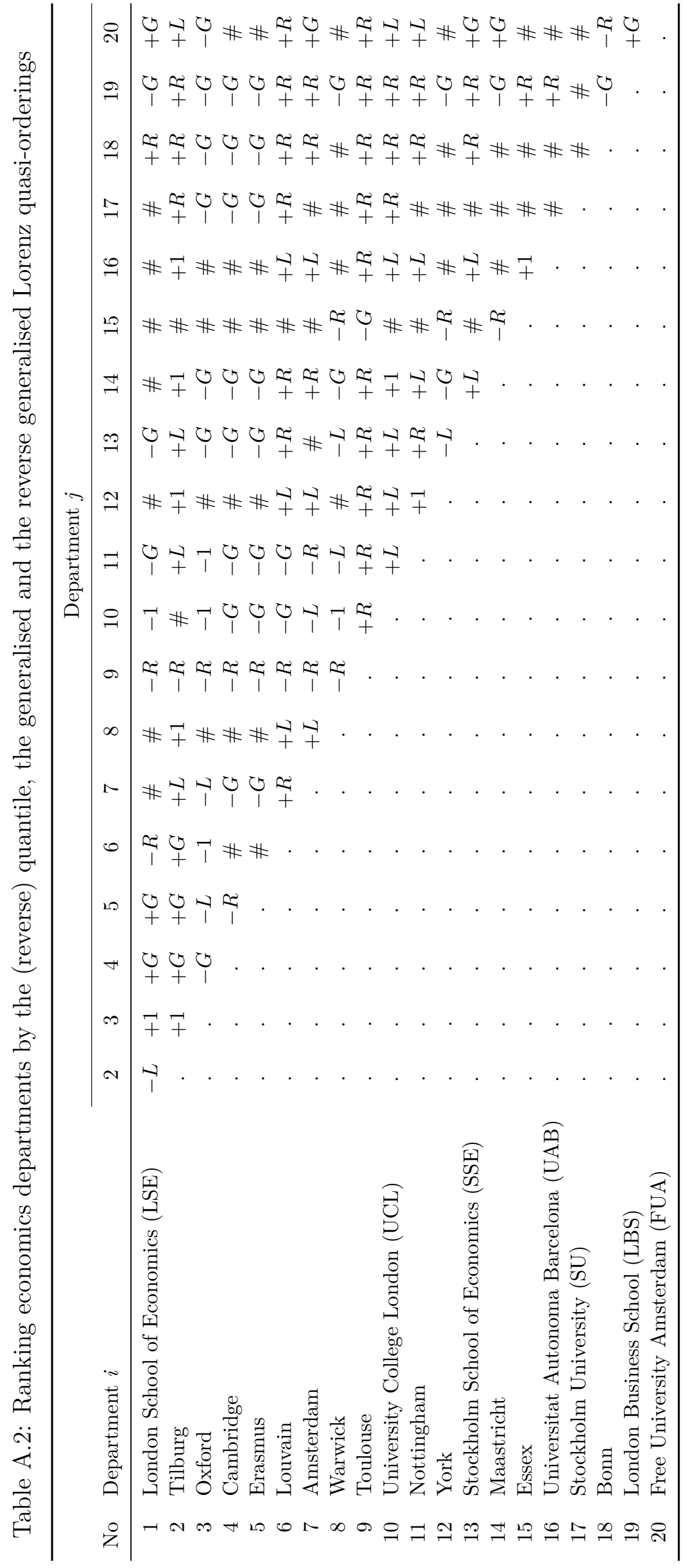




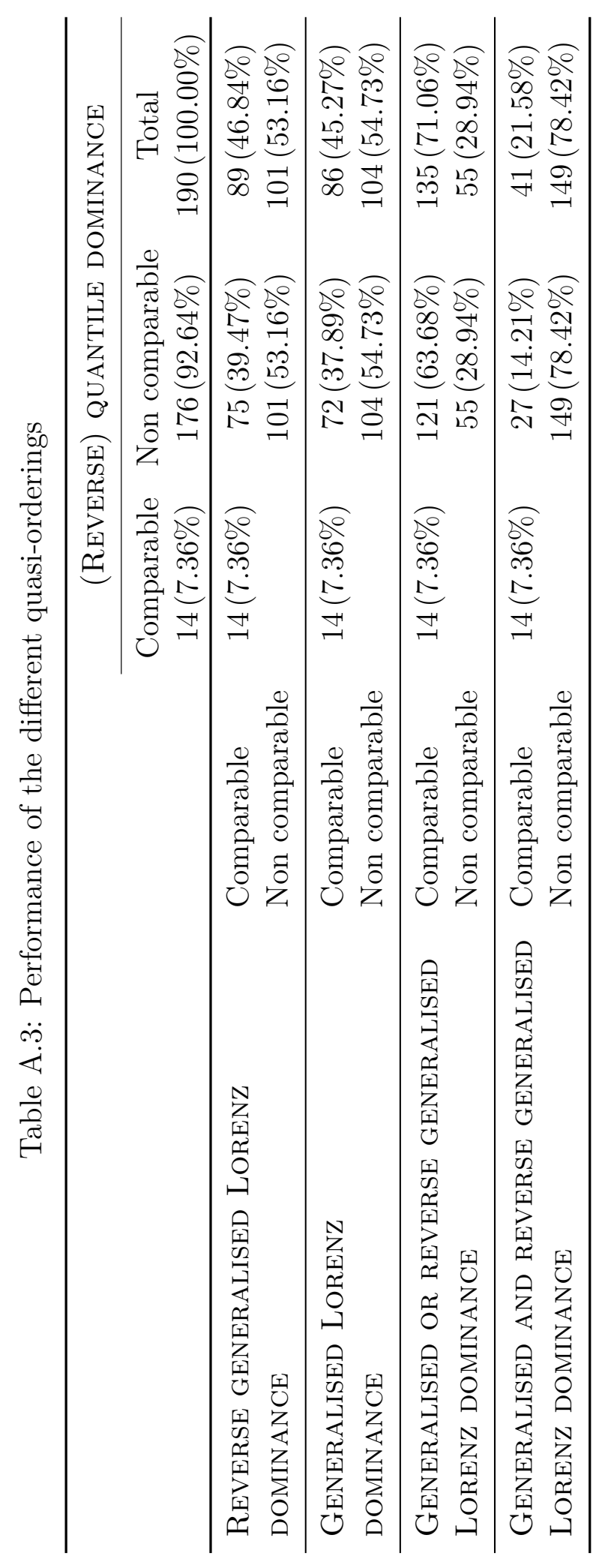




\section{References}

Atkinson, A. B. and Bourguignon, F. (1987). Income distributions and differences in needs. In G. Feiwel, editor, Arrow and the Foundations of the Theory of Economic Policy, pages 350-370. Macmillan, New-York.

Atkinson, A. B., Rainwater, L., and Smeeding, T. (1995). Income Distribution in OECD Countries. OECD Social Policy Studies No. 18, Paris.

Bazen, S. and Moyes, P. (2003). International comparisons of income distributions. Research on Economic Inequality, 9, 85-111.

Blundell, R. and Lewbel, A. (1991). The information content of equivalence scales. Journal of Econometrics, 50, 49-68.

Bourguignon, F. (1989). Family size and social utility: Income distribution dominance criteria. Journal of Econometrics, 42, 67-80.

Buhmann, B., Rainwater, L., Schmaus, G., and Smeeding, T. M. (1988). Equivalence scales, well-being, inequality and poverty: Sensitivity estimates across ten countries using the Luxembourg Income Study (LIS) database. Review of Income and Wealth, 34, 115-142.

Carayol, N. and Lahatte, A. (2009). Dominance relations and universities ranking. GREThA Discussion Paper \#2009-02, CNRS-Université Montesquieu Bordeaux 4.

Chateauneuf, A. and Moyes, P. (2004). Lorenz non-consistent welfare and inequality measurement. Journal of Economic Inequality, 2, 61-87.

Chateauneuf, A. and Moyes, P. (2006). Measuring inequality without the Pigou-Dalton condition. In M. McGillivray, editor, Inequality, Poverty and Well-Being, pages 22-65. Palgrave Macmillan, Basingtoke, Hampshire/New-York.

Combes, P.-P. and Linnemer, L. (2003). Where are the economists who publish? Publication concentration and rankings in Europe based on cumulative publications. Journal of the European Economic Association, 1, 1250-1308.

Coupé, T. (2003). Revealed performances: Worldwide rankings of economists and economics departments, 1990-2000. Journal of the European Economic Association, 1, 1309-1345.

Dalton, H. (1920). The measurement of the inequality of incomes. Economic Journal, 30, $348-361$.

Dusansky, R. and Vernon, C. J. (1998). Rankings of U.S. economics departments. Journal of Economic Perspectives, 12, 157-170.

Ebert, U. (1988). Measurement of inequality: An attempt at unification and generalization. Social Choice and Welfare, 5, 59-81.

Ebert, U. and Moyes, P. (2010). Talents, preferences and inequality of well-being. GREThA, Université Montesquieu Bordeaux IV.

Ebert, U. and Moyes, P. (2011). Inequality of well-being and isoelastic equivalence scales. GREThA, Université Montesquieu Bordeaux IV.

Fishburn, P. C. (1974). Convex stochastic dominance with continuous distribution functions. Journal of Economic Theory, 7, 143-158.

Fishburn, P. C. and Vickson, R. G. (1978). Theoretical foundations of stochastic dominance. 
In G. Whitmore and M. Findlay, editors, Stochastic Dominance, pages 39-113. Lexington Books, Lexington, MA.

Foster, J. E. and Shorrocks, A. F. (1988). Poverty orderings and welfare dominance. Social Choice and Welfare, 5, 91-110.

Gastwirth, J. (1971). A general definition of the Lorenz curve. Econometrica, 39, 1037-1039.

Gravel, N. and Moyes, P. (2008). Bidimensional inequalities with an ordinal variable. GREThA Discussion Paper 2008-14, Université Montesquieu Bordeaux IV.

Hardy, G. H., Littlewood, J. E., and Pólya, G. (1952). Inequalities. Cambridge University Press, Cambridge, second edition.

Kalaitzidakis, P., Mamuneas, T., and Stengos, T. (1999). European economics: An analysis based on publications in core journals. European Economic Review, 43, 1150-1168.

Kalaitzidakis, P., Mamuneas, T., and Stengos, T. (2003). Rankings of academic journals and institutions in economics. Journal of the European Economic Association, 1, 1346-1366.

Kolm, S.-C. (1976). Unequal inequalities I. Journal of Economic Theory, 12, 416-442.

Lubrano, M. and Protopopescu, C. (2004). Density inference for ranking european research systems in the field of economics. Journal of Econometrics, 123, 345-369.

Lubrano, M., Bauwens, L., Kirman, A. P., and Protopopescu, C. (2003). Ranking european economic departments: A statistical approach. Journal of the European Economic Association, 1, 1367-1401.

Magdalou, B. and Moyes, P. (2009). Deprivation, welfare and inequality. Social Choice and Welfare, 32, 253-273.

Makdissi, P. and Wodon, Q. (2004). Robust comparisons of natural resources depletion indices. Economics Bulletin, 9, 1-9. http://www.economicsbulletin.com/2004/volume9/EB04I30002A.pdf.

Marshall, A. W. and Olkin, I. (1979). Inequalities: Theory of Majorization and its Applications. Academic Press, New-York.

Moyes, P. and Shorrocks, A. F. (1994). Transformations of stochastic orderings. In W. Eichhorn, editor, Models and Measurement of Welfare and Inequality, pages 162-175. Springer Verlag, Berlin.

Quiggin, J. (1993). Generalized Expected Utility Theory. The Rank-Dependent Model. Kluwer Academic Publishers, Boston/Dordrecht/London.

Riese, M. and Brunner, J. K. (1998). Measuring the severity of unemployment. Journal of Economics, 67, 167-180.

Ross, S. M. (1983). Stochastic Processes. John Wiley, New-York.

Saposnik, R. (1981). Rank-dominance in income distributions. Public Choice, 36, 147-151.

Sen, A. K. (1976). Poverty: An ordinal approach to measurement. Econometrica, 44, 219-231.

Sen, A. K. (1992). Inequality Reexamined. Clarendon Press, Oxford.

Shaked, M. and Shanthikumar, G. J. (1994). Stochastic Orders and their Applications. Academic Press, Oxford.

Shorrocks, A. F. (1983). Ranking income distributions. Economica, 50, 3-17. 
Shorrocks, A. F. and Foster, J. E. (1987). Transfer sensitive inequality measures. Review of Economic Studies, 54, 485-497.

Wong, W.-K. and Li, C.-K. (1999). A note on convex stochastic dominance. Economics Letters, 62, 293-300.

Yaari, M. E. (1987). The dual theory of choice under risk. Econometrica, 55, 99-115.

Yaari, M. E. (1988). A controversial proposal concerning inequality measurement. Journal of Economic Theory, 44, 381-397. 